\title{
Generalized Lusin sets with the Blackwell property
}

by

R. M. Shortt * (Middletown, Conn.) and K.P.S. Bhaskara Rao (Calcutta)

Abstract. A separable space $(X, \mathscr{B})$ has the Blackwell property if whenever $\mathscr{C}$ is a countably generated sub- $\sigma$-algebra of $\mathscr{B}$ separating points of $X$, then $\mathscr{C}=\mathscr{B}$. We characterize which Lusin and Sierpiński sets have the Blackwell property and consider when the property is preserved under the taking of products.

§ 0. Introduction. At the 1983 Oberwolfach Conference on Measure Theory, the first author posed the problem of whether the construction of universally null Blackwell spaces is possible within ZFC. At present, the matter does not seem to have found resolution. However, Jakub Jasiński [7] in Gdańsk has very recently shown that under the assumption of $\mathrm{CH}$, there are some Lusin sets with the Blackwell property and some without. As he points out, his arguments apply also to their category analogue, the Sierpiński sets.

The present paper is an effort to unify these results with other theorems about Blackwell spaces in a rather general framework. Many examples of Blackwell spaces may then be seen to arise as generalized Lusin sets with respect to a $\sigma$-ideal of Borel sets. Vary the $\sigma$-ideal, and different species of Blackwell spaces emerge.

Our point of departure remains the observation that every set is a generalized Lusin set with respect to some Borel $\sigma$-ideal. For each fixed $\sigma$-ideal, only the "larger" Lusin sets enjoy the Blackwell pioperty, and the strong Blackwell property is reserved for those stiil even larger. Further gradations of size may be observed for sets various products of which are also Blackwell.

Section 1 defincs Blackwell properties and introduces the concept of a Lusin set with respect to a $\sigma$-ideal, as well as the notion of size ( $\mathscr{F}$-density) mentioned above. Section 2 goes on to characterize $\mathscr{I}$-Lusin sets with Blackwell and strong Blackwcll properties for an arbitrary $\sigma$-ideal (Proposition. 2). It is secn that if $\mathscr{I}$ is a uniformisable $\sigma$-idecl, then for $\mathscr{I}$-Lusin sets, these properties actually coincide.

* The first author has been supported in this research by a National Science Foundation grant DMS-8412413. 
Propositions 3 and 4 take up the matter of Blackwell properties for spaces of the form $X^{n} \times S$, where $X$ is an $\mathscr{I}$-Lusin set and $S$ is a standard space.

Section 3 presents proofs (assuming CH) that there are $\mathscr{I}$-Lusin sets with and without Blackwell properties (Propositions 6 and 7). Proposition 5 guarantees the existence of $\mathscr{I}$-Lusin sets of any prescribed order of density. Section 4 takes on the $\sigma$-ideal of countable sets. This $\sigma$-ideal turns out to be uniformisable, with the consequence that for spaces with totally imperfect complement, Blackwell and strong Blackwell properties coincide. This result was obtained earlier by the first author [18], but here it is viewed as part of a general theory.

Section 5 defines the notion of a uniformisable set. Like the Blackwell spaces, such sets turn out to be a generalization of analytic spaces (Proposition 10), and they have a close relationship with the Blackwell problem (Proposition 13).

The rest of the paper specializes to the $\sigma$-ideal of measure zero sets. In this case, the $\mathscr{I}$-Lusin sets are precisely the classical Sierpiński sets. This $\sigma$-ideal is not uniformisable (Lemma 13), and indeed, Blackwell and strong Blackwell properties part company for such spaces, at least under $\mathrm{CH}$ (Proposition 18). Interestingly enough, the construction also solves an open problem of probabilistic measure theory posed by Ramachandran [14]: the notions of independent random variable due to Steinhaus and Kolmogoroff do not necessarily agree on Blackwell domains.

Section 7 takes up the question of Blackwe properties for various products of Sierpiński sets. The structure of the $\sigma$-ideal of measure zero sets is sufficiently rich so as to allow a fuller analysis than that given in Section 2, e.g. Proposition 19. Some work of Kellerer on marginal problems puts in an unexpected guest appearance at this juncture.

Section 8 obtains a pair of measurable selection theorems (Propositions 23 and 25) using disintegration of measures, hyperspaces of compact sets and Strassen's Theorem from probability in concert. With these results, it becomes possible to establish another characterization of Sierpiński sets with Blackwell properties (Proposition 26). This theorem finds employment in Section 9, where it is used to prove Proposition 27 on Blackwell properties for countable unions of such sets.

Section 10 considers the question of whether $X \times S$ is Blackwell whenever $S$ is standard and $X$ is a strongly Blackwell Sierpiński set. The issue remains unresolved, but the question is shown to bc equivalent to a conjecture about measurable selections and doubly-stochastic mcasures (Proposition 29).

Thanks are due to Jakub Jasiński, Henryk Leszczyński, Janusz Pawlikowski, and Czesław Ryll-Nardzewski, who found errors in earlier draughts of this work

§1. Preliminaries. For the elements of descriptive set theory and basic results about Borel structures, the reader is referred to [1] and [9]. The notation and terminology of this paper generally follow [1].

A separcble space is a measurable (Borel) space $(X, \mathscr{B})$ whose $\sigma$-algebra $\mathscr{B}$ is countably generated (c.g.) and contains all singletons drawn from the set $X$. If $\mathscr{C}$ is a sub- $\sigma$-algebra of $\mathscr{B}$, and $A \subset X$, then the notation $\mathscr{C}(A)=\{C \cap A: C \in \mathscr{C}\}$ will be used to denote the relative $\mathscr{E}$-structure on $A$. A separable space $(S, \mathscr{B})$ is standard (resp. analytic) if there is a complete (resp. analytic) separable metric topology on $S$ for which $\mathscr{B}$ is the corresponding Borel structure.

A separable space $(X, \mathscr{B})$ has the Blackwell property if whenever $\mathscr{C}$ is a c. g. sub$\sigma$-algebra of $\mathscr{B}$ separating points of $X$, then necessarily $\mathscr{C}=\mathscr{B}$. Say that $(X, \mathscr{B})$ has the strong Blackwell property if whenever $\mathscr{C} \subset \mathscr{D}$ are c.g. sub- $\sigma$-algebras of $\mathscr{B}$ with the same atoms, the necessarily $\mathscr{C}=\mathscr{D}$. For equivalent definitions, consult [1].

Throughout this paper, $(S, \mathscr{B})$ will denote a fixed uncountable standard space. Often, the space will be denoted simply as $S$. A $\sigma$-ideal $\mathscr{I}$ of sets in $\mathscr{B}(S)$ is continuous if it contains all singleton subsets of $S$. Let $\mathscr{I}$ be any $\sigma$-ideal in $\mathscr{B}(S)$. (The set $S$ is never a member of $\mathscr{I}$.) A subset $X$ of $S$ is $\mathscr{I}$-Lusin if $X$ is uncountable and the intersection of $X$ with every member of $\mathscr{I}$ is countable.

We shall often consider the $n$-fold product of $S$ with itself as a standard space, denoted by $S^{n}$. Here $n$ may be any positive integer. If $N_{1}, \ldots, N_{n}$ are subsets of $S$, then $\left\langle N_{1}, \ldots, N_{n}\right\rangle$ is the set of all $\left(s_{1}, \ldots, s_{n}\right)$ in $S^{n}$ such that for some $k$, one has $s_{k} \in N_{k}$. Thus $\left\langle N_{l}, . ., N_{n}\right\rangle$ is the complement in $S^{n}$ of $N_{1}^{c} \times N_{2}^{c} \times . . \times N_{n}^{c}$. A subset $R$ of $S^{n}$ is $\mathscr{I}$-reticulate in $S^{n}$ if these are sets $N_{1}, \ldots, N_{n}$ in $\mathscr{I}$ with $R \subset\left\langle N_{1}, \ldots, N_{n}\right\rangle$. A subset of $T$ of $S \times S$ is an $\mathscr{I}$-thread if

1) $T$ is the graph of a Borel-isomorphism between sets $A$ and $B$ in $\mathscr{B}(S)$;

2) $T$ is not $\mathscr{I}$-reticulate.

A subset $\mathscr{G}$ of $S \times S$ is an $\mathscr{I}$-graph if

1) $\mathscr{G}$ is the graph of some Borel function $g: A \rightarrow S$, where $A \in \mathscr{B}(S)$;

2) $\mathscr{G}$ is not $\mathscr{I}$-reticulate.

Note. The set $\mathscr{G}$ is allowed to be of either form $\{(s, t): t=g(s)\}$ or $\{(s, t): s=g(t)\}$. A $\sigma$-ideal $\mathscr{I}$ is uniformisable if every set $R$ in $\mathscr{B}(S \times S)$ which is not $\mathscr{I}$-reticulate contains an $\mathscr{I}$-thread.

A subset $X$ of $S$ is $\mathscr{I}$-dense (of order 1 ) in $S$ if every $B \in \mathscr{B}(S)$ disjoint from $X$ is member of $\mathscr{I}$. Say that $X$ is $\mathscr{I}$-dense of order $n$ in $S$ if $X^{n}$ intersects every $R$ in $\mathscr{B}\left(S^{n}\right)$ which is not $\mathscr{I}$-reticulate. It is easy to see that for each $n \geqslant 1, \mathscr{I}$-density of order $n+1$ implies $\mathscr{I}$-density of order $n$.

One particular type of $\sigma$-ideal in $\mathscr{B}(S)$ will prove to be of special interest. Let $X$ be a fixed uncountable subset of $S$. Define $\mathscr{F}(X)$ to be the $\sigma$-ideal of all $B$ in $\mathscr{B}(S)$ with $B \cap X$ countable. Then $\mathscr{I}(X)$ is a continuous $\sigma$-ideal, and $X$ is $\mathscr{I}(X)$-dense in $S$. In fact, one may easily prove.

LEMMA 1. Let $\mathscr{I}$ be a $\sigma$-ideal in $\mathscr{B}(S)$ and let $X$ be an uncountable subset of $S$.

1) The set $X$ is $\mathscr{I}$-Lusin in $S$ if and only if $\mathscr{I} \subset \mathscr{I}(X)$.

2) If $\mathscr{I}(X) \subset \mathscr{I}$, then $X$ is $\mathscr{I}$-dense in $S$. The converse holds if $\mathscr{I}$ is assumed to be continuous.

Thus, $\mathscr{I}(X)$ is the largest $\sigma$-ideal $\mathscr{I}$ in $\mathscr{B}(S)$ for which $X$ is $\mathscr{I}$-Lusin, whilst $\mathscr{I}(X)$ is the smallest continuous $\sigma$-ideal $\mathscr{I}$ for which $X$ is $\mathscr{I}$-dense. 
Suppose $\mathscr{I}$ is a given $\sigma$-ideal in $\mathscr{B}(S)$ and that $\mathscr{C}$ and $\mathscr{D}$ are c.g. sub- $\sigma$-algebras of $\mathscr{B}(S)$. Say that $\mathscr{C}$ is $\mathscr{I}$-proper in $\mathscr{D}$ if

1) $\mathscr{C} \subset \mathscr{D}$;

2) if $N \in \mathscr{I}$, then $\mathscr{C}\left(N^{c}\right) \neq \mathscr{D}\left(N^{c}\right)$.

Given Marczewski functions $f$ and $g$ for countable generators of $\sigma$-algebras $\mathscr{Q}^{\prime}$ and $\mathscr{D}$, we define the set

$$
T(\mathscr{C}, \mathscr{D})=\{(s, t) \in S \times S: f(s)=f(t) \quad \text { and } \quad g(s) \neq g(t)\} .
$$

The definition of $T(\mathscr{C}, \mathscr{D})$ does not depend on the particular choice of the Marczewski functions $f$ and $g$.

LEMMA 2. Let $\mathscr{C} \subset \mathscr{D}$ be c.g. sub- $\sigma$-algebras of $\mathscr{B}(S)$. Then the following are equivalent:

1) $\mathscr{C}$ is $\mathscr{I}$-proper in $\mathscr{D}$

2) there is some set $D$ in $\mathscr{D}$ not equivalent to any, $\mathscr{C}$-set modulo $\mathscr{I}$ : in other words, $D \triangle C \notin \mathscr{I}$ for any $C$ in $\mathscr{C}$

3) $T(\mathscr{C}, \mathscr{D})$ is not $\mathscr{I}$-reticulate in $S \times S$.

Proof. 1) $\Rightarrow 2$ ): We proceed to prove the contrapositive and assume that no set $D$ as in 2) exists. Let $D_{1}, D_{2}, \ldots$ be a countable generator for $\mathscr{D}$. Then there is a sequence $C_{1}, C_{2}, \ldots$ in $\mathscr{C}$ with $C_{n} \sim D_{n} \bmod (\mathscr{P})$. Put

$$
N=\cup\left(D_{n} \triangle C_{n}\right)
$$

a set in $\mathscr{I}$. Then $\mathscr{C}\left(N^{c}\right)=\mathscr{D}\left(N^{c}\right)$, since for each $n, D_{n} \cap N^{c}=C_{n} \cap N^{c}$. Thus $\mathscr{C}$ is not $\mathscr{I}$-proper in $\mathscr{D}$.

2) $\Rightarrow 1$ ): Suppose $D$ in $\mathscr{D}$ is as specified and that $N \in \mathscr{I}$. Then for no set $C$ in $\mathscr{C}$ does it happen that $D \cap N^{c}=C \cap N^{c}$. If this were true, then $C \triangle D \subset N$, a contradiction.

3) $\Rightarrow 1$ ): Suppose that $N \in \mathscr{I}$ is such that $\mathscr{C}\left(N^{c}\right)=\mathscr{D}\left(N^{c}\right)$. Then one sees that $T(C, \mathscr{D}) \subset\langle N, N\rangle$.

1) $\Rightarrow 3)$ : Suppose that these are sets $N_{1}$ and $N_{2}$ in $\mathscr{I}$ with $T(\mathscr{C}, \mathscr{D}) \subset\left\langle N_{1}, N_{2}\right\rangle$. Put $N=N_{1} \cup N_{2}$. Then $\mathscr{C}\left(N^{c}\right) \subset \mathscr{D}\left(N^{c}\right)$ have the same atoms. Since every standard set is strongly Blackwell $\left[1 ;\right.$ p. 21], we have $\mathscr{C}\left(N_{\mathscr{s}}^{c}\right)=\mathscr{D}\left(N^{c}\right)$, as desired.

Say that a subset $X$ of $S$ satisfies condition $(J)$ or $(J+)$ for the $\sigma$-ideal $\mathscr{I}$ if

(J) whenever $\mathscr{C}$ is a c.g. sub- $\sigma$-algebra of $\mathscr{B}(S)$ which is $\mathscr{I}$-proper in $\mathscr{B}(S)$, then there is some $\mathscr{C}$-atom $C$ such that $C \cap X$ contains at least two points (i.e. $\mathscr{C}$ does not separate points of $X$ );

$(\mathrm{J}+) \quad$ whenever $\mathscr{C}$ and $\mathscr{D}$ are c.g. sub- $\sigma$-algebras of $\mathscr{B}(S)$ with $\mathscr{C} \mathscr{I}$-proper in $\mathscr{D}$ then there is some $\mathscr{C}$-atom $C$ such that $C \cap X$ contains two points separated by $\mathscr{D}$.

Compare the definition of $(*)$-Blackwell conditions in [18], as well as their use for a special case in [7] ("Jasiński conditions"). Condition ( $\mathrm{J}+$ ) guarantees that whenever $\mathscr{C}$ is very much smaller than $\mathscr{D}$ (with respect to $\mathscr{P}$ ), then the set $X$ contains witnesses to this fact.

Another sort of $\sigma$-ideal on which our attention will focus consists of sets which are null with respect to a given probability measure. Let $m$ be a Borel probability measure on $S$. Define $\mathscr{I}(m$ to be the collection of all $m$-null sets in $\mathscr{B}(S)$. The $\sigma$-ideal $\mathscr{I}(m)$ is continuous if and only if $m$ is a continuous measure. Mcst often, we shall take $m$ to be continuous. A subset $X$ of $S$ is $\mathscr{I}(m)$-dense in $S$ if and only if $m^{*}(X)=1$. If $m$ is continuous, then the $\mathscr{I}(m)$-Lusin sets are the classical sets with property $(S)$ studied by Sierpiński [2], [20]. We shall often use the terms $\mathscr{F}(m)$-Lusin and $m$-Sierpiński interchangeably.

The following result will be quite handy in the sequel:

LEMMA 3. Let $m$ be an atomless probability on $S$. If $B$ is a set in $\mathscr{B}\left(S^{2}\right)$ of positive $(m \otimes m)$-area, then $B$ contains an $\mathscr{I}(m)$-thread.

Note. The result also holds whenever $m$ is purely atomic, but not if $m$ is of mixed type.

Proof. By the usual isomorphism theorems, it is no loss of generality to take $m$ to be Lebesgue measure on the interval $S=10,1[$. For each real $c$, define $B(c)=\{(s, t) \in B: t=s+c\}$. Then by Fubini's Theorem (at a $45^{\circ}$ angle), at least one of the sets $B(c)$ has positive linear measure. Such a set $B(c)$ is an $\mathscr{I}(m)$-thread.

Consider the following restriction on a $\sigma$-ideal $\mathscr{I}$ :

(R) Whenever $B_{1}$ and $B_{2}$ are two members of $\mathscr{B}(S) \backslash \mathscr{I}$, then the rectangle $B_{1} \times B_{2}$ contains an $\mathscr{I}$-thread.

LEMMA 4. Let $\mathscr{I}$ be a continuous $\sigma$-ideal in $\mathscr{B}(S)$ satisfying restriction (R). If $X \subset S$ satisfies (J), then $X$ is $\mathscr{I}$-dense. In particular, this implication holds when $\mathscr{F}$ is uniformisable.

Proof. If $X$ is not dense in $S$, then there is some set $B \subset S \backslash X$ in $\mathscr{B} \backslash \mathscr{I}$. Since $\mathscr{I}$ is continuous, $B$ decomposes into two (necessarily uncountable) disjoint sets $B_{1}$ and $B_{2}$ in $\mathscr{B} \backslash \mathscr{I}$. Applying restriction (R), we find an $\mathscr{I}$-thread $G$ inside $B_{1} \times B_{2}$. Now $G$ is the graph of a Borel-isomorphism $g$ between sets $C_{1}$ and $C_{2}$ in $\mathscr{B}(S)$. Define $f: S \rightarrow S$ by the rule

$$
f(s)= \begin{cases}g(s), & s \in C_{1}, \\ s, & s \notin C_{1}\end{cases}
$$

Define $\mathscr{C}=\mathscr{B}(f)=\left\{f^{-1}(B): B \in \mathscr{B}(S)\right\}$. We claim that $\mathscr{C}$ is $\mathscr{I}$-proper in $\mathscr{B}(S)$. For each $N \in \mathscr{I}$, there is in $G$ a point $(s, g(s))$ not in $(N \times S) \cup(S \times N)$. Then $s$ and $g(s)$ are points of $N^{c}$ not separated by $\mathscr{C}$. So $\mathscr{C}\left(N^{c}\right) \neq \mathscr{B}\left(N^{c}\right)$.

However, $\mathscr{C}$ separates points of $X$. The Lemma follows by contraposition.

We shall see later that a $\sigma$-ideal may satisfy restriction $(R)$ but not be uniformisable. This will be the case with the $\sigma$-ideal of null sets for a continuous measure (see Section 6). Also, it is not true that every continuous $\sigma$-ideal $\mathscr{I}$ satisfies restriction (R). An example to illustrate the point will be given in Section 6 . 
§ 2. Results for general $\sigma$-ideals. As usual, $\mathscr{I}$ is taken to be a $\sigma$-ideal in the standard structure $\mathscr{B}(S)$.

Proposition 1. Suppose $X$ is $\mathscr{I}$-dense in $S$.

A) If $X$ is $\mathscr{I}$-dense of order 2 in $S$, then $X$ satisfies condition ( $\mathrm{J}+)$.

B) If $X$ satisfies condition $(\mathrm{J}+)$, then $X \times X$ intersects every $\mathscr{I}_{- \text {graph in }} S \times S$.

C) If $X$ satisfies condition (J), then $X \times X$ intersects every $\mathscr{I}$-thread in $S \times S$.

Proof. Part A. As noted in Lemma $2, \mathscr{C}$ proper in $\mathscr{D}$ means that $T(\mathscr{C}, \mathscr{D})$ is not $\mathscr{I}$-reticulate. If $X$ is dense of order 2 , then $(X \times X) \cap T(\mathscr{C}, \mathscr{D}) \neq \varnothing$. So there are points $x$ and $x^{\prime}$ in $X$ not separated by $\mathscr{C}$ but separated by $\mathscr{D}$. This establishes. condition $(\mathrm{J}+)$.

Part B. We assume that $S=] 0,1[$ under the usual linear order and Borel structure. The contrapositive will be proved.

Begin by taking $\mathscr{G}$ to be an $\mathscr{I}$-graph with $\mathscr{G} \cap(X \times X)=\varnothing$. We shall assume that there is some $A$ in $\mathscr{B}(S)$ and a Borel function $k: A \rightarrow S$ such that $\mathscr{G}=\operatorname{graph}(k)$. $=\{(s, k(s)): s \in A\}$. This assumption is made without loss of generality. Define

$$
\begin{aligned}
\Delta & =\{(s, s): s \in S\}, \\
\Delta_{+} & =\{(s, t): s<t\}, \\
\Delta_{-} & =\{(s, t): s>t\}
\end{aligned}
$$

as subsets of $S \times S$. Since $X$ is $\mathscr{I}$-dense in $S$, it follows that $\mathscr{G} \cap \Delta$ is $\mathscr{I}$-reticulate.

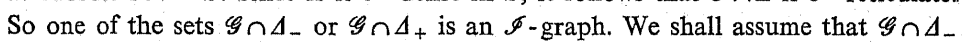
is an $\mathscr{I}$-graph. The other case, where it is $\mathscr{G} \cap \Delta_{+}$that is an $\mathscr{I}$-graph involves an argument entirely parallel to the one we now offer.

With these assumptions in place, there is some $\varepsilon>0$ such that $\mathscr{G} \cap \Delta_{-}(\varepsilon)$ is. an $\mathscr{I}$-graph, where

$$
\Delta_{-}(\varepsilon)=\{(s, t) \in S \times S: s-\varepsilon>t\} .
$$

Also, there is some open interval $\mathcal{O}$ of length $\varepsilon$ such that $\mathscr{G}_{\cap} \Delta_{-}(\varepsilon) \cap(\mathcal{O} \times S)$ is. an $\mathscr{F}$-graph. This set is the graph of a Borel function $h$ defined on a set $D$ in $\mathscr{B}(S)$ :

$$
\mathscr{G} \cap \Delta_{-}(\varepsilon) \cap(\mathcal{O} \times S)=\{(s, h(s)): s \in D\} .
$$

Now, whenever $s$ and $t$ are elements of $D$, then $h(s)<s-\varepsilon<t$, so that $h(D) \cap D=\varnothing$. Define functions $f$ and $g$ on $S$ by the formulae

$$
\begin{aligned}
& f(s)= \begin{cases}h(s), & s \in D, \\
s, & s \in D^{c}\end{cases} \\
& g(s)= \begin{cases}(h(s), 0), & s \in D^{c} \\
(s, s), & s \in D^{c}\end{cases}
\end{aligned}
$$

Here, $g$ takes values in $\boldsymbol{R}^{2}$. Let $\mathscr{C}$ and $\mathscr{D}$ be the sub- $\sigma$-algebras generated by $f$ and $g$, respectively.
We claim that $\mathscr{C}$ is $\mathscr{I}$-proper in $\mathscr{D}$. This will follow from a proof that $T(\mathscr{C}, \mathscr{D})$ is not $\mathscr{I}$-re.iculate. For this, we need only note that

$$
T(\mathscr{C}, \mathscr{D})=\{(s, h(s)): s \in D\} \cup\{(h(t), t): t \in D\} .
$$

On the other hand, $T(\mathscr{C}, \mathscr{D}) \cap(X \times X)=\mathscr{Q}$, so that $\mathscr{C}(X)$ and $\mathscr{D}(X)$ have the same. atoms. Thus condition $(\mathrm{J}+)$ fails for $X$.

Part C. Essentially the same proof as for part $B$, taking $\mathscr{G}$ to be an $\mathscr{I}$-thread. and noting that, in this case, $\mathscr{D}$ as defined above equals $\mathscr{B}(S)$.

Proposition 2. Let $X$ be an $\mathscr{I}$-Lusin subset of $S$.

1) If $X$ satisfies $(\mathrm{J}+)$, then $X$ is strongly Blackwell. The converse obtains if $X$ is $\mathscr{I}$-dense in $S$.

2) If $X$ satisfies (J), then $X$ is Blackwell. The converse obtains if $X$ is $\mathscr{I}$-dense in $S$.

Proof. We show that if $X$ satisfies condition ( $\mathrm{J}+$ ), then $X$ is strongly Blackwell. The implication $(\mathrm{J}) \rightarrow$ Blackwell runs parallel and its proof is omitted.

So suppose that $\mathscr{C}(X) \subset \mathscr{D}(X)$ are c.g. sub- $\sigma$-algebras of $\mathscr{B}(X)$ with the same atoms. Then there are c.g. $\sigma$-algebras $\mathscr{C} \subset \mathscr{D} \subset \mathscr{B}(S)$ whose relativizations to $X$ are $\mathscr{C}(X)$ and $\mathscr{D}(X)$. Condition $(\mathrm{J}+)$ implies that $\mathscr{C}$ is not proper in $\mathscr{D}$. Therefore, there is some set $N$ in $\mathscr{I}$ with $\mathscr{C}\left(N^{\mathcal{C}}\right)=\mathscr{D}\left(N^{c}\right)$. Since $X$ is an $\mathscr{I}$-Lusin set, it follows that $X \cap N$ is countable.

Let $A$ be the union of all $\mathscr{C}$-atoms $C$ with the property that $C \cap X \cap N \neq \varnothing$. There are only countably many such $\mathscr{C}$-atoms, so that $A \in \mathscr{C}(S)$. Since $\mathscr{C}(X \cap A)$. and $\mathscr{D}(X \cap A)$ have the same countable set of atoms, one has $\mathscr{C}(X \cap A)=\mathscr{D}(X \cap A)$. Now $X \cap A^{c} \subset N^{c}$, so that $\mathscr{C}\left(X \cap A^{c}\right)=\mathscr{D}\left(X \cap A^{c}\right)$. Given $D$ in $\mathscr{D}$, there are $\mathscr{C}$ - sets $C_{1}$ and $C_{2}$ such that

$$
\begin{aligned}
D \cap X & =(D \cap X \cap A) \cup\left(D \cap X \cap A_{2}^{c}\right) \\
& =\left(C_{1} \cap X \cap A\right) \cup\left(C_{2} \cap X \cap A^{c}\right)
\end{aligned}
$$

is a member of $\mathscr{C}(X)$. So $\mathscr{C}(X)=\mathscr{D}(X)$, as desired.

To conclude the proof, we show that if $X$ is $\mathscr{I}$-dense and strongly Blackwell, then $X$ satisfies condition $(\mathrm{J}+)$. Again, the implication Blackwell $\rightarrow(\mathrm{J})$ is similar and so its proof is omitted.

So suppose that $\mathscr{C}$ and $\mathscr{D}$ are c.g. sub- $\sigma$-algebras of $\mathscr{B}(S)$ with $\mathscr{C}$ proper in $\mathscr{D}$. We shall assume that $(\mathrm{J}+)$ fails and derive a contradiction. If $(\mathrm{J}+)$ fails, then $\mathscr{C}(X) \subset \mathscr{D}(X)$ have the same atoms. From the strong Blackwell property, $\mathscr{C}(X)=\mathscr{D}(X)$. Let $g$ be a Marczewski function for $\mathscr{D}(S)$. Then when restricted to $X, g$ generates $\mathscr{D}(X)=\mathscr{C}(X)$. Call this restricted function $g_{0}$. Since it is. $\mathscr{C}(X)$-measurable, it extends to a $\mathscr{C}$-measurable function $f$ on $S$. Put

$$
B=\{s \in S: f(s)=g(s)\} .
$$

Then $B \in \mathscr{B}(S)$; in fact, $B \in \mathscr{D}$.

Since $X \subset B$, we know that $S \backslash B \in \mathscr{I}$. We claim that $\mathscr{C}(B)=\mathscr{D}(B)$. For, suppose, 
that $b$ and $b^{\prime}$ are points in $B$ separated by $\mathscr{D}$. Then $g(b) \neq g\left(b^{\prime}\right)$. Thus $f(b) \neq f\left(b^{\prime}\right)$ and so $b$ and $b^{\prime}$ are separated by $\mathscr{C}$. This proves the claim.

A contradiction has been established: $\mathscr{C}$ is proper in $\mathscr{D}$, but $\mathscr{C}(B)=\mathscr{D}(B)$

with $S \backslash B \in \mathscr{I}$.

Remark. It should be noted that in the proof of the converse, the hypothesis that $X$ is $\mathscr{I}$-Lusin was not used.

CoRollary. Suppose that $\mathscr{I}$ is a uniformisable $\sigma$-ideal. Let $X$ be an $\mathscr{I}$-Lusin set $\mathscr{I}$-dense in $S$. Then the following are cquivalent:

1) $X$ is dense of order 2 ;

2) $X$ is strongly Blackweil

3) $X$ is Blackwell.

Note. The implication 1$) \Rightarrow 2$ ) does not require $\mathscr{I}$ to be uniformisable or $X$ to be $\mathscr{I}$-dense; 3$) \Rightarrow 1$ ) does not require $\mathscr{I}$ to be continuous or $X$ to be $\mathscr{I}$-Lusin; 2) $\Rightarrow 3$ ) needs no conditions on $\mathscr{I}$ or $X$.

Definition. A subsct $X$ of $S$ is analytically $\mathscr{I}$-dense (of order 1) in $S$ if for each analytic subset $A$ of $S \backslash X$, there is some set $N$ in $\mathscr{I}$. with $A \subset N$.

Clearly, this notion is stronger than that of simple $\mathscr{I}$-density. The following is immediate:

LEMMA 5. A subset $X$ of $S$ is analytically $\mathscr{I}(X)$-dense if and only if Borel sets separate $X$ from every analytic $A$ with $A \cap X=\varnothing$.

EXAMPLE 1. Let $A$ be an analytic subset of $S$. Then $A$ is analytically $\mathscr{I}(A)$-dense in $S$. This is Lusin's first separation principle in another costume.

EXAMPLE 2. Let $X$ be a co-analytic subset of $S$. Then $X$ is analytically $\mathscr{I}(X)$ dense in $S$ if and only if $X$ is a Borel set.

Say that a subset $X$ of $S$ is analytically $\mathscr{I}$-dense of order $n$ in $S$ if $X^{n}$ intersects every analytic set $A \subset S^{n}$ which is not $\mathscr{I}$-reticulate in $S^{n}$. In other words, if $A \subset S^{n} \backslash X^{n}$, then there are sets $N_{1}, \ldots, N_{n}$ in $\mathscr{I}$ with $A \subset\left\langle N_{1}, \ldots, N_{n}\right\rangle$.

It is easy to show that analytic $\mathscr{I}$-density of order $n+1$ implies that of order $n$ for $n=1,2, \ldots$

Lemma 6. Let $X$ be an $\mathscr{I}$-Lusin subset of $S$. The following statements are equivalent:

1) $X$ is analytically $\mathscr{I}$-dense of order $n$;

2) whenever $A$ is an analytic subset of $S^{n} \backslash X^{n}$, then there are $\mathscr{I}$-sets $N_{1}, \ldots, N_{n}$ contained in $S \backslash X$ such that $A \subset\left\langle N_{1}, \ldots, N_{n}\right\rangle$.

Proof. The implication $2 \Rightarrow 1$ is immediate The proof of $1 \Rightarrow 2$ proceeds via induction on $n$. For $n=1$, we see that if $A \subset S \backslash X$ is analytic, then there is some $N$ in $\mathscr{I}$ with $A \subset N$. But since $X$ is $\mathscr{I}$-Lusin, we know that $N \cap X$ is countable. So $A \subset N \backslash X \subset S \backslash X$ fills the bill.

Now we assume the result for all dimensions less than $n(n \geqslant 2)$ and suppose that $X$ is analytically $\mathscr{I}$-dense of order $n$. Suppose that $A \subset S^{n} \backslash X^{n}$ is an analytic set. There are sets $N_{1}, \ldots, N_{n}$ in $\mathscr{I}$ with $A \subset\left\langle N_{1}, \ldots, N_{n}\right\rangle$. Since $X$ is $\mathscr{I}$-Lusin, the set $N_{1} \cap X$ may be listed as $x_{1}, x_{2}, \ldots$ For each $i \geqslant 1$, the section $A\left(x_{i}\right)$ of $A$ over $x_{i}$ is an analytic subset of $S^{n-1} \backslash X^{n-1}$. By the induction hypcthesis, there arc $\mathscr{I}$-sets $N_{2}^{(i)}, \ldots, N_{n}^{(i)}$ contained in $S \backslash X$ with $A\left(x_{i}\right)=\left\langle N_{2}^{(i)}, \ldots, N_{n}^{(i)}\right\rangle$. For $2 \leqslant k \leqslant n$, put $N_{k}^{\prime}=N_{k} \cup N_{k}^{(1)} \cup N_{k}^{(2)} \cup$.. Then $A \subset\left\langle N_{1} \backslash X, N_{2}^{\prime}, N_{3}^{\prime}, \ldots, N_{n}^{\prime}\right\rangle$.

Repeat the procedure for each of the other $n-1$ co-ordinates in $S^{i}$. This will produce $\mathscr{I}$-sets $M_{1}, \ldots, M_{n}$ contained in $S \backslash X$ such that $A \subset\left\langle M_{1}, \ldots, M_{n}\right\rangle$, is desired.

Proposition 3. Let $\mathscr{I}$ be a $\sigma$-ideal in the standard structure $\mathscr{O}(S)$ and suppose that $X \subset S$ is an $\mathscr{I}$-Lusin set analytically $\mathscr{I}$-dense of order $2 n(n \geqslant 1)$. Then $X^{n} \times S$ is a strongly Blackwell space.

Proof. It suffices, by Propositions 1 and 2, to prove that $X^{n} \times S$ is $\mathscr{I}\left(X^{n} \times S\right)$-dense of order 2 in $S^{n} \times S$. So suppose $R$ is a member of $\mathscr{B}\left(S^{n} \times S \times S^{n} \times S\right)$ contained in $\left(S^{n} \times S \times S^{n} \times S\right)\left(X^{n} \times S \times X^{n} \times S\right)$. Let $f: S^{n} \times S \times S^{n} \times S \rightarrow S^{n} \times S^{n}$ be defined as the projection

$$
f\left(s_{1}, \ldots, s_{n}, s_{n+1}, t_{1}, \ldots, t_{n}, t_{n+1}\right)=\left(s_{1}, \ldots, s_{n}, t_{1}, \ldots, t_{n}\right) .
$$

Then $f(R)$ is an analytic subset of $\left(S^{n} \times S^{n}\right) \backslash\left(X^{n} \times X^{n}\right)$. Since $X$ is analytically $\mathscr{F}$-dense of order $2 n$, there are $\mathscr{I}$-sets $A_{1}, \ldots, A_{1}$ and $B_{1}, \ldots, B_{n}$ contained in $S \backslash X$ (Lemma 6) with

and $\mathrm{s}$

$$
f(R) \subset\left\langle A_{1}, . ., A_{n}, B_{1}, \ldots, B_{n}\right\rangle,
$$

$$
R \subset\left\langle\left\langle A_{1}, \ldots, A_{n}, \varnothing\right\rangle,\left\langle B_{1}, \ldots, B_{n}, \varnothing\right\rangle\right\rangle .
$$

Since $\left\langle A_{1}, \ldots, A_{n}, \varnothing\right\rangle$ and $\left\langle B_{1}, \ldots, B_{n}, \varnothing\right\rangle$ do not intersect $X^{n} \times S$, we sce that $R$ is $\mathscr{I}\left(X^{n} \times S\right)$-reticulate, as desired.

PROPOSITION 4. Let $\mathscr{I}$ be a $\sigma$-ideal in the standird Borel structure $B(S)$ and suppcse that $X \subset S$ is analytically $\mathscr{I}$-dense (order 1 ) in $S$. For $n \geqslant 1$, if $X^{n} \times S$ is Blackwell, then $X$ is analytically $\mathscr{I}$-dense of order $2 n$ in $S$.

Proof. Suppose that $A$ is an analytic subset of $S^{2 n} \backslash X^{2 n}$. There is some Borel mapping $h$ of $S$ onto $A$. We define a Borel subset $T$ of $\left(S^{n} \times S\right) \times\left(S^{n} \times S\right)$ as

$$
\begin{aligned}
T & =\left\{\left(s_{1}, \ldots, s_{n}, s_{n+1}, t_{1}, \ldots, t_{n}, t_{n+1}\right): s_{n+1}=t_{n+1} \quad\right. \text { and } \\
h\left(s_{n+1}\right) & \left.=\left(s_{1}, \ldots, s_{n}, t_{1}, \ldots, t_{n}\right)\right\} .
\end{aligned}
$$

The set $T$ is disjoint from $\left(X^{n} \times S\right) \times\left(X^{i 1} \times S\right)$. Furthermore, each section of $T$ (either direction) over a point in $S^{n} \times S$ is at most singleton.

Now since $X^{n} \times S$ is Blackwell, it follows (Propositions 1 and 2) that $\left(X^{n} \times S\right)$ $\times\left(X^{n} \times S\right)$ meets every $\mathscr{I}\left(X^{n} \times S\right)$-thread in $\left(S^{n} \times S\right) \times\left(S^{n} \times S\right)$. Since the one-one graph $T$ is not met, it must be that there is some $N$ in $\mathscr{I}\left(X^{n} \times S\right)$ with $T \subset\langle N, N\rangle$.

2 - Fundamenta Mathematicae 127. 
Let $f$ be the natural projection map from $S^{n} \times S$ onto $S^{n}$. Then $M=f(N)$ is an analytic subset of $S^{n}$ such that

a) $M \cap X^{n}$ is countable;

b) $A \subset\langle M, M\rangle$.

We have proved that to each analytic $A \subset S^{2 n} \backslash X^{2 n}$ there corresponds at least one analytic $M \subset S^{n}$ with properties a) and b).

The proof now proceeds by induction on $n$. For $n=1$, take $M$ corresponding to $A$ as above. Since $X$ is analytically $\mathscr{I}$-dense in $S$, there is some $\mathscr{I}$-set $N$ with the result has been estab$M \subset N$. Then $A \subset\langle N, N\rangle$ as desired. Now we assume that the result has been established for dimensions $k \leqslant n$ and consider the case for $n+1$. Since $X^{n+1} \times S$ is Blackwell, so too is $X^{n} \times S$, so that (by the induction hypothesis) $X$ is analytically $\mathscr{I}$-dense wello of order $n+1$. Again, given $A \subset S^{2(n+1)} \backslash X^{2(n+1)}$, take $M \subset S^{n+1}$ corresponding to $A$ as before. There are $\mathscr{I}$-sets $N_{1}, \ldots, N_{n+1}$ with $M \subset\left\langle N_{1}, \ldots, N_{n+1}\right\rangle$. So

$$
A \subset\left\langle N_{1}, \ldots, N_{n+1}, N_{1}, \ldots, N_{n+1}\right\rangle
$$

as desired.

COROLlaRY. Let $X \subset S$ be an $\mathscr{I}$-Lusin set analytically $\mathscr{I}$-dense in $S$ and suppose that $A$ is an uncountable analytic subset of $S$. Then for each $n \geqslant 1$, the following are equivalent:

1) $X^{n} \times S$ is strongly Blackwell

2) $X^{n} \times A$ is strongly Blackwell;

3) $X^{n} \times A$ is Blackwell;

4) $X^{n} \times S$ is Blackwell;

5) $X$ is analytically $\mathscr{I}$-dense of order $2 n$ in $S$.

Proof. The implications $1 \Rightarrow 2$ and $3 \Rightarrow 4$ follow from the fact that $A$ is a measurable image of $S$, and that $S$ is isomorphic to a Borel subset of $A$. The implication $2 \Rightarrow 3$ is obvious, $4 \Rightarrow 5$ is Proposition 4 , whilst $5 \Rightarrow 1$ is Proposition 3 .

\$ 3. Existence theorems. In this section, we make use of the continuum hypothesis $(\mathrm{CH})$ to prove the existence of $\mathscr{I}$-Lusin sets with and without Blackwell properties. Later, through the use of Proposition 5 (regarding the existence of $\mathscr{I}$-Lusin sets of any prescribed density) we shall demonstrate the existence of sets $\mathscr{I}$-Lusin sets of any prescribed density) we shack $X^{n+1} \times S$ is not Blackwell:

LEMMA $7(\mathrm{CH})$. Let $\mathscr{I}$ be a continuous $\sigma$-ideal in $\mathscr{B}(S)$. Let $n$ be a positive integer and suppose that $\mathscr{A}$ is a collection of subset; of $S^{n}$ such that

1) no sct in $\mathscr{A}$ is $\mathscr{I}$-reticulate;

2) for each $A$ in $\mathscr{A}$ and $N$ in $\mathscr{I}$, the set $A \backslash\langle N, \ldots, N\rangle$ is a member of $\mathscr{A}$ Suppose that $Y \subset S$ is such that $Y^{n}$ intersects every set in $\mathscr{A}$. Then there is an $\mathscr{I}$-Lusin $X \subset Y$ with the same property.

Proof. Well-order the members of $\mathscr{A}$ and of $\mathscr{I}$ in transfinite series $A_{0} A_{1} A_{2} \ldots A_{\alpha} \ldots$ and $N_{0} N_{1} N_{2} \ldots N_{\alpha} \ldots, \alpha<\kappa_{1}$. Select points $y_{1}^{(0)}, \ldots, y_{n}^{(0)}$ in $Y$ with $\left(y_{1}^{(0)}, \ldots, y_{n}^{(0)}\right) \in A_{0} \backslash\left\langle N_{0}, \ldots, N_{0}\right\rangle$. For each $\alpha$, put $M_{\alpha}=\bigcup\left\{N_{\beta}: \beta \leqslant \alpha\right\} \cup$ $\cup\left\{y_{i}^{(\beta)}: 1 \leqslant i \leqslant n\right.$ and $\left.\beta<\alpha\right\}$ and choose points $y_{1}^{(\alpha)}, \ldots, y_{n}^{(\alpha)}$ in $Y$ with

$$
\left(y_{1}^{(\alpha)}, \ldots, y_{n}^{(\alpha)}\right) \in A_{\alpha} \backslash\left\langle M_{\alpha}, \ldots, M_{\alpha}\right\rangle .
$$

Properties of $\mathscr{A}$ ensure the possibility of such selections. Define

$$
X=\left\{y_{i}^{(\alpha)}: 1 \leqslant i \leqslant n \text { and } \alpha<\aleph_{1}\right\} \text {. }
$$

This set has the desired properties.

Proposition $5(\mathrm{CH})$. Let $\mathscr{I}$ be a continuous $\sigma$-ideal in $\mathscr{B}(S)$ and let $n$ be a positive integer. Suppose that $Y \subset S$ has one of the following properties:

1) $Y \times Y$ intersects every $\mathscr{I}$-thread in $S \times S$;

2) $Y \times Y$ intersects every $\mathscr{I}$-graph in $S \times S$;

3) $Y$ is $\mathscr{I}$-dense of order $n$ in $S$;

4) $Y$ is analytically $\mathscr{I}$-dense of order $n$ in $S$.

Then there is an $\mathscr{I}$-Lusin set $X \subset Y$ with the same property.

Indication. This is a simple application of Lemma 7: let $\mathscr{A}$ be either the collection of $\mathscr{I}$-threads, $\mathscr{I}$-graphs, or Borel or analytic subsets of $S^{n}$. which are not $\mathscr{I}$-reticulate.

Proposition $6(\mathrm{CH})$. Let $\mathscr{I}$ be a continuous $\sigma$-ideal in $\mathscr{B}(S)$. Then there is an $\mathscr{I}$-Lusin subset $X$ of $S$ with the strong Blackwell property.

Proof. From Proposition 5 with $Y=S$, we find an $\mathscr{I}$-Lusin set $X$ which is $\mathscr{I}$-dense of order 2 in $S$. The result follows from Propositions 1 and 2.

Proposition $7(\mathrm{CH})$. Let $\mathscr{I}$ be a continuous $\sigma$-ideal in $\mathscr{B}(S)$ satisfying restriction (R). There is an $\mathscr{I}$-Lusin subset $X$ of $S$ which is dense but lacks the Blackwell property.

Proof. Since the $\sigma$-ideal $\mathscr{I}$ is continuous, there are disjoint sets $B_{1}$ and $B_{2}$ in $\mathscr{B}(S) \backslash \mathscr{I}$. Restriction (R) implies that $B_{1} \times B_{2}$ contains a thread $G$. There are sets $C_{1}$ and $C_{2}$ in $\mathscr{B}(S) \backslash \mathscr{I}$ so that $G$ is the graph of a Borel-isomorphism $g$ of $\mathrm{C}_{1}$ onto $C_{2}$. Define $f: S \rightarrow S$ by the rule

$$
f(s)= \begin{cases}g(s), & s \in C_{1}, \\ g^{-1}(s), & s \in C_{2}, \\ s, & s \in S \backslash\left(C_{1} \cup C_{2}\right) .\end{cases}
$$

Note that $g(s) \neq s$ for any $s$ in $C_{1}$ and that $f=f^{-1}$

Let $B_{0} B_{1} B_{2} \ldots B_{\alpha} \ldots(\alpha<c)$ be a listing in transfinite series of all members of $\mathscr{B}(S) \backslash \mathscr{I}$ and let $N_{0} N_{1} N_{2} \ldots N_{\alpha} \ldots(\alpha<c)$ be a similar listing of $\mathscr{I}$. For each countable ordinal $\alpha$, put $M_{\alpha}=\bigcup\left\{N_{\beta}: \beta \leqslant \alpha\right\}$.

Choose $x_{0}$ from $B_{0} \backslash M_{0}$ and for each $\alpha<c$ select $x_{\alpha}$ from

$$
B_{\alpha} \backslash\left(M_{\alpha} \cup\left\{f\left(x_{\beta}\right): \beta<\alpha\right\}\right) \text {. }
$$


Put $X=\left\{x_{\alpha}: \alpha<c\right\}$. Then $X$ is $\mathscr{I}$-Lusin and dense in $S$. But $X \times X$ does not intersect $G$, so that from Proposition 1, condition (J) fails for $X$. Proposition 2 shows that $X$ lacks the Blackwell property.

$\S 4$. Borcl-density. Let $\mathscr{I}$ be the $\sigma$-ideal of all countable subsets of the uncountable standard space $S$. Then $\mathscr{I}$ is continuous, and the $\mathscr{I}$-Lusin sets are precisely the uncountable subsets of $S$. The notions of $\mathscr{I}$-reticulate sets, $\mathscr{I}$-thread, and $\mathscr{I}$-density for this $\sigma$-ideal coincide with those of "reticulate set", "thread", and "Borel-density" as used in [17], [18], and [19].

LeMma 8. The $\sigma$-ideal $\mathscr{I}$ is uniformisable.

Indication. This follows from results in [5], as well as an argument in Sarbadhikari's note [15].

The following was obtained in [18]. It is now seen to be a consequence of our general theory, in particular the corollary to Proposition 2.

Proposition 8. Let $X$ be a subset of an uncountable Polish space $S$ such that $S \backslash X$ is totally imperfect. Then the following are equivalent:

1) $X$ is Blackwell;

2) $X$ is strongly Blackwell;

3) $X$ is $\mathscr{I}$-dense ("Borel-dense" in [17]) of order 2.

COROLlarY. If $X$ is a Blackwell subset of $S$ with totally imperfect complement, then any $Y$ such that $X \subset Y \subset S$ is strongly Blackwell.

\$ 5. Uniformisable sets. Let $X$ be a fixed uncountable subset of $S$. Define $\mathscr{I}(X)$ to be the $\sigma$-ideal consisting of all $B$ in $\mathscr{B}(S)$ with $B \cap X$ countable. Then $\mathscr{I}(X)$ is continuous, and $X$ is $\mathscr{I}(X)$-dense of order 1 in $S$.

LEMMA 9. Suppose that $X$ is $\mathscr{I}(X)$-dense of order 2 in $S$. Then the sets $R$ in $\mathscr{B}(S \times S)$ which are not $\mathscr{I}(X)$-reticulate are precisely those for which $R \cap(X \times X)$ is not contained in a countable union of horizontal and vertical sections.

Proof. Clearly, any $R$ with this property is not $\mathscr{I}(X)$-reticulate. Conversely, if $R \cap(X \times X)$ is contained in a countable union of sections, then by removing these sections from $R$, we obtain a Borel set $R_{0}$ disjoint from $X \times X$. Second-order density implies that $R_{0}$ is $\mathscr{I}(X)$-reticulate. So also is $R$.

Proposition 9. Let $A$ be an cinulytic slibset of $S$. Then $A$ is $\mathscr{I}(A)$-dense of order 2 in $S$.

Proof. Let $R$ be a member of $\mathscr{B}(S \times S)$ disjoint from $A \times A$. Then $R_{1}=R \cap(S \times A)$ is an analytic set whose projection onto the first factor is disjoint from $A$. Lusin's first separation principle implies that there is some $N_{1} \in \mathscr{B}(S)$ such that $N_{1} \cap A=\varnothing$ and $R_{1} \subset\left(N_{1} \times S\right)$ Now $R_{2}=R \backslash\left(N_{1} \times S\right)$ is a member of $\mathscr{B}(S \times S)$ whose projection onto the second factor does not meet $A$. Thus there is some $N_{2} \in \mathscr{B}(S)$ such that $N_{2} \cap A=\varnothing$ and $R_{2} \subset\left(S \times N_{2}\right)$. Then $N=N_{1} \cup N_{2} \in \mathscr{I}(A)$, and $R \subset(N \times S) \cup(S \times N)$.

Propositron 10. If $A$ is analytic, then the $\sigma$-ideal $\mathscr{I}(A)$ is uniformisable.
Proof. Suppose that $R$ is a member of $\mathscr{B}(S \times S)$ not $\mathscr{I}(A)$-reticulate. From Proposition 9 and Lemma 9 , we know that $R \cap(A \times A)$ is not contained in a countable union of sections. It follows from [5; Theorem 4.4] or [15] that $R \cap(A \times A)$ contains an uncountable standard set $T$ each of whose horizontal and vertical sections is at most a singleton. See also the discussion in [19]. Thus $T$ is an $\mathscr{I}(A)$-thread.

If $X$ is any uncountable subset of $S$, then certainly $X$ is an $\mathscr{T}(X)$-Lusin set. However, there may be a great many other $\mathscr{I}(X)$-Lusin sets essentially larger than $X$.

LEMMA 10. Lat $A$ be an anclytic subset of $S$. Ther the $\mathscr{F}(A)$-Lusin sets are those uncountable subsets $Y$ of $S$ whose intersection with each constituent of the co-cinalytic set $S \backslash A$ is countable.

Proof. If $X$ is an $\mathscr{I}(A)$-Lusin set, and $C$ is a constituent of $S \backslash A$, then $C \in \mathscr{I}(A)$, so that $X \cap C$ is countable. On the other hand, suppose $X$ intersects each constituent of $S \backslash A$ in a countable set. Given $N$ in $\mathscr{I}(A)$, we know that $N \cap A$ is countable, so that $N \backslash A$ is Borel and contained in a countable union of constituents (boundedness theorem). This forces $X \cap N$ to be countable.

Proposition 11. Let $A$ be an analytic subset of $S$ and suppose that $Y$ is a set whose intersection with each constituent of $S \backslash A$ is countable. Then $A \cup Y$ is strongly, Blackwell.

Remark. Jakub Jasiński has recently [7] used Martin's Axiom to obtain a partial converse to this result.

Proof. From Proposition 9, the sets $A$ and $A \cup Y$ are $\mathscr{I}(A)$-dense of order 2. Lemma 10 says that $A \cup Y$ is $\mathscr{I}(A)$-Lusin. The result now follows from the corollary to Proposition 2.

The following result has been obtained independently by Jasiński [7]. It follows easily from the theory developed here, and we include it for the sake of completeness.

Proposition 12. Let $X$ be an uncountable Blackwell (resp. strongly Blackwell) subset of $S$. If $Y$ is an $\mathscr{I}(X)$-Lusin set, then $X \cup Y$ is Blackwell (resp. strongly Blackwell).

Proof. Clearly, $X \cup Y$ is $\mathscr{I}(X)$-Lusin and $\mathscr{I}(X)$-dense in $S$. If $X$ is Blackwell (resp. strongly Blackwell), then from Proposition 2, $X$ and $X \cup Y$ satisfy condition (J), (resp. condition $(\mathrm{J}+)$ ). Another application of Proposition 2 does the job.

Say that a subset $X$ of an uncountable standard space $S$ is a uniformisable set if $\mathscr{F}(X)$ is uniformisable. We have shown that every analytic set is uniformisable. Thus, the notion of uniformisable space generalizes and properly contains that of analytic space. To see this, note that if $S \backslash X$ is totally imperfect ( $X$ is Borel-dense), then $\mathscr{I}(X)$ is the $\sigma$-ideal of countable subsets of $S$. So from Lemma $8, X$ is uniformisable.

Proposition 13. Let $X$ be a subset of $S$ which is Blackwell, but not strongly Blackwell. Then $X$ not a uniformisable set.

Proof. As mentioned, $X$ is certainly an $\mathscr{I}(X)$-Lusin set $\mathscr{I}(X)$-dense of order 1 . The result is immediate from Propositions 1 and 2. 
Blackwell spaces without the strong Blackwell property have been constructed under the axioms $\mathrm{CH}$ and $\mathrm{MA}+$ not $-\mathrm{CH}$. We shall give another construction $(\mathrm{CH})$ is section 6. The situation in ZFC is still not known. See [4] and the discussion in [1].

$\S 6$. Sierpiński sets. Throughout this section we let $m$ denote a continuous probability on the Borel structure $\mathscr{B}(S)$. Also, $\mathscr{I}=\mathscr{I}(m)$ will be the $\sigma$-ideal of all $m$-null sets in $\mathscr{B}(S)$. Then $\mathscr{I}$ is continuous, and the $\mathscr{I}$-Lusin subsets of $S$ are the classical Sierpiński sets [2]. A subset $X$ of $S$ is $\mathscr{G}$-dense if and only if $m^{*}(X)=1$.

We shall prove that $\mathscr{I}$ satisfies restriction (R) but is not uniformisable. Additionally, $\mathscr{I}$ will be used to construct a continuous $\sigma$-ideal violating restriction (R). To facilitate discussion, it becomes convenient to introduce the following set function, defined on subsets $R$ of $S \times S$ :

$$
\mu R=\inf \{m A+m B: R \subset(A \times S) \cup(S \times B) ; A, B \in \mathscr{B}(S)\} .
$$

Then $\mu$ is a Carathéodory outer measure with very few $\mu$-measurable sets.

LEMMA 11. A set $R \subset S \times S$ is $\mathscr{I}$-reticulate if and only if $\mu R=0$.

Proof. One direction (only if) is obvious. To prove the converse, suppose that $\mu R=0$. Choose sets $A_{n}$ and $B_{n}$ in $\mathscr{B}(S)$ with $R \subset\left(A_{n} \times S\right) \cup\left(S \times B_{n}\right)$ and $m\left(A_{n}\right)+$ $+m\left(B_{n}\right)<2^{-n}$. Put $A=\limsup A_{n}$ and $B=\limsup B_{n}$. Then $R \subset(A \times S) \cup(S \times B)$, and from the Borel-Cantelli Lemma, $m A=m B=0$. So $R$ is reticulate.

Proposition 14. Suppose that $(S \times S) \backslash R$ is a countable union of Borel rectangles (e.g. if $S$ is metric and $R$ is closed). Then $\mu R \geqslant \varepsilon$ if and only if there is a measure $v$ on $\mathscr{B}(S \times S)$ both of whose marginals equal $m$ such that $\mu R \geqslant \varepsilon$.

Proof. This is essentially a result of Strassen [21], discussed and extended for the measurable setting in [16].

LEMMA 12. The $\sigma$-ideal $\mathscr{I}$ satisfies restriction (R)

Proof. Suppose that $B_{1}$ and $B_{2}$ are sets in $\mathscr{B}(S)$ with $m\left(B_{1}\right) m\left(B_{2}\right)$ positive. Then there are sets $C_{1} \subset B_{1}$ and $C_{2} \subset B_{2}$ in $\mathscr{B}(S)$ with $m\left(C_{1}\right)=m\left(C_{2}\right)>0$. Alsol there is a Borel-isomorphism $f$ of $C_{1}$ onto $C_{2}$ preserving $m$-measure: for all Bore, $B \subset C_{1}$, one has $m f(B)=m(B)$. The graph of $f$ is a thread inside $B_{1} \times B_{2}$.

LEMMA 13. The $\sigma$-ideal $\mathscr{I}(m)$ is not uniformisable.

Proof. Let $f$ be a Borel-isomorphism of $S$ onto $S \times S$ mapping $m$ onto the product measure $m \otimes m$. Let $p: S \times S \rightarrow S$ be projection onto the first co-ordinate and define $g: S \rightarrow S$ to be the composition $g=p \circ f$. Then $G=\operatorname{graph}(g)$ is nonreticulate in $S \times S$ but contains no threads.

To see this, suppose that $G \subset\left(N_{1} \times S\right) \cup\left(S \times N_{2}\right)$ with $m\left(N_{1}\right)=m\left(N_{2}\right)=0$. Then $S \subset N_{1} \cup g^{-1}\left(N_{2}\right)$, but $\quad m g^{-1}\left(N_{2}\right)=m f^{-1} p^{-1}\left(N_{2}\right)=(m \otimes m) p^{-1}\left(N_{2}\right)$ $=m\left(N_{2}\right)=0$, a contradiction. So $G$ is not reticulate.

On the other hand, suppose that $T$ is a thread in $G$. Then $T$ is the graph of the restriction of $g$ to some $C$ in $\mathscr{B}(S)$. On $C$, the function $g$ is one-one. So $f(C)$ is a subset of $S \times \dot{S}$ each of whose vertical sections is at most singleton. So $m(C)=(m \otimes m) f(C)=0$, a contradiction.

Let $\mathscr{J}$ be an arbitrary $\sigma$-ideal in $\mathscr{B}(S)$. Say that a subset $R$ of $S \times S$ is $(\mathscr{F}, \mathscr{J})$-reticulate if $R$ can be covered by a set of the form $\left(N_{1} \times S\right) \cup\left(S \times N_{2}\right)$ with $m\left(N_{1}\right)=0$ and $N_{2} \in \mathscr{J}$.

LEMMA 14. A subset $R$ of $S \times S$ is not $(\mathscr{F}, \mathscr{J})$-reticulate if and only if there is some $\varepsilon>0$ such that for all $B$ in $\mathscr{B}(S)$ with $m(B) \geqslant 1-\varepsilon$, the set $R \cap(B \times S)$ is not $(\mathscr{F}, \mathscr{J})$-reticulate.

Proof. One direction (if) is obvious. To prove the converse, we suppose that for each $n$, there is some $B_{n}$ in $\mathscr{B}(S)$ with $m\left(B_{n}\right) \geqslant 1-2^{-n}$ such that $R \cap\left(B_{n} \times S\right)$ is $(\mathscr{I}, \mathscr{J})$-reticulate. Thus there are sets $N_{n}$ and $M_{n}$ with $R \cap\left(B_{n} \times S\right) \subset\left(N_{n} \times S\right) \cup$ $\cup\left(S \times M_{n}\right)$ and $m\left(N_{n}\right)=0$ and $M_{n} \in \mathscr{J}$. Put $A_{n}=S \backslash B_{n}$ and note that

$$
R \subset\left(\left(A_{n} \cup N_{n}\right) \times S\right) \cup\left(S \times M_{n}\right) .
$$

Define $A=\limsup \left(A_{n} \cup N_{n}\right)$ and $M=\cup M_{n}$. Then $R \subset(A \times S) \cup(S \times M)$ and one has $M \in \mathscr{J}$ and, by the Borel-Cantelli lemma, $m(A)=0$. Thus $R$ is $(\mathscr{I}, \mathscr{J})$-reticulate.

EXAMPLE. Not every continuous $\sigma$-ideal satisfies restriction (R.).

Construction. Realize $S$ as the union of the intervals $I_{1}=[0,1]$ and $I_{2}=[2,3]$ as a compact metric space under the usual structure. Define a $\sigma$-ideal $\mathscr{N}$ in $\mathscr{B}(S)$ to be the collection of all Borel sets of the form $N_{1} \cup N_{2}$, where $N_{1}$ is a subset of $I_{1}$ of Lebesgue measure $m\left(N_{1}\right)=0$ and $N_{2}$ is a subset of $I_{2}$ of first Baire category.

We shall prove that $I_{1} \times I_{2}$ contains no $\mathscr{N}$-threads. Suppose for the sake of contradiction that $T$ were such a thread. Then $T$ is the graph of some Borel-isomorphism $f$. Let $\varepsilon$ be as specified in Lemma 14 for the set $T$ any apply Lusin's Theorem to produce a compact $K \subset I_{1}$ with $m(K) \geqslant 1-\varepsilon$ and such that $f$ is continuous on $K$ (in fact, a homeomorphism). Now from Lemma $14, T \cap\left(K \times I_{2}\right)$ is a thread; it is the graph of the restriction of $f$ to $K$, which function we call $g$.

The set $K$ decomposes into a union $K=N \cup F$, where $m(N)=0$ and $F$ is of first category in $K$. Since $g$ is a homeomorphism, $g(F)$ is of first category in $g(K)$ and hence also in $I_{2}$. Thus $T \cap\left(K \times I_{2}\right)=\operatorname{graph}(g) \subset(N \times S) \cup(S \times g(F))$ is $\mathscr{N}$-reticulate. This gives the desired contradiction.

Jasiński [7] has, under the assumption of $\mathrm{CH}$, constructed a Sierpiński set $X_{1}$ such that if $X_{2} \supset X_{1}$ is another Sierpinski set, then automatically $X_{2}$ is strong Blackwell. In his example, $m^{*}\left(X_{1}\right)=1$. We shall see that such behavior is actually typical.

Proposition $15(\mathrm{CH})$. There are Sierpinski subsets of $S$ with the strong Blackwell property.

Proof. Apply Proposition 6.

Proposition $16(\mathrm{CH})$. There are Sierpinski subseis of $S$ without the Blackwell property.

Proof. Apply Proposition 7 and Lemma 12. 
Remark. Jasiński shows [7] without $\mathrm{CH}$ that if there is a Sierpiński set, then there is some Sierpiński set without the Blackwell property.

Proposition 17. Suppose that $X_{1}$ and $X_{2}$ are $\mathscr{F}(m)$-Lusin sets (" $m$-Sierpiniski sets") with $X_{1} \subset X_{2}$ and $m^{*}\left(X_{1}\right)=m^{* *}\left(X_{2}\right)$.

1) If $X_{1}$ is Blackwell, then $X_{2}$ is Blackwell;

2) If $X_{1}$ is strongly Blackwell, then $X_{2}$ is strongly Blackwell.

Proof. Choose $B \supset X_{2}$ with $B \in \mathscr{B}(S)$ and $m(B)=m^{*}\left(X_{2}\right)$. We know that $m^{*}\left(X_{2}\right)>0$, so that $n=m / m(B)$ is a continuous probability on the standard space $(B, \mathscr{B}(B))$. Since $X_{1}$ is $\mathscr{I}(n)$-dense in $B$, Proposition 2 will apply to prove the results.

However, for the $\sigma$-ideal $\mathscr{I}=\mathscr{I}(\mathrm{m})$, the conditions in Proposition 1 are not equivalent. The nonuniformisability of $\mathscr{I}$ exerts great influence in the following.

PRoposition $18(\mathrm{CH})$. Let $m$ be a continuous probability on a standard space $S$. Then $S$ contains an $\mathscr{I}(m)$-Lusin set $X$ which is Blackwell, but not strongly Blackwell.

Proof. Any two standard, continuous probability spaces $\left(S_{1}, m_{1}\right),\left(S_{2}, m_{2}\right)$ are isomorphic, so it suffices to work with a particular instance of such a space. Put $S=\left[0,1[\times] 0,1 l\right.$ under the usual Borel structure. Put $\left.A_{1}=\{0\} \times\right] 0,1[$ and $\left.A_{2}=S \backslash A_{1}=\right] 0,1[\times] 0,1[$. Define a Borel probability $m$ on $S$ as follows:

1) on subsets of $A_{1}, m$ agrees with one half the usual linear Lebesgue measure;

2) on subsets of $A_{2}, m$ agrees with one half the usual planar area measure. We define functions $f$ and $g$ on $S$ as follows:

a) $f(x, y)=y$;

b) $g(x, y)=y+\operatorname{sgn}(x)$.

Let $\mathscr{C}$ and $\mathscr{D}$ be the $\sigma$-algebras on $S$ generated by $f$ and $g$, respectively. Clearly $\mathscr{C} \subset \mathscr{D}$.

We claim that $\mathscr{C}$ is $\mathscr{I}(m)$-proper in $\mathscr{D}$. To see this, suppose that $N$ is a null set for $m$. Then $f\left(A_{1} \cap N\right)$ is a linear null set in ]0, 1[, and from Fubini's Theorem, almost all horizontal sections of $A_{2} \cap N$ have linear measure zero. It follows that there is some $y$ in $] 0,1[$ such, that

1) $(0, y) \in N$;

2) $(x, y) \in N$ for some $x>0$.

The points $(0, y)$ and $(x, y)$ are not separated by $\mathscr{C}$, but are separated by $\mathscr{Q}$. Thus $\mathscr{C}\left(N^{\mathfrak{C}}\right) \neq \mathscr{D}\left(N^{\mathrm{C}}\right)$, and $\mathscr{C}$ is proper in $\mathscr{Q}$.

It follows that the set $T(\mathscr{C}, \mathscr{D})$ is not $\mathscr{I}(m)$-reticulate. A pair $(s, t)$ belongs to $T(\mathscr{C}, \mathscr{D})$ if and only if $s$ and $t$ are members of the same horizontal $y$-section of $S$, and one of the two points is of the form $(0, y)$. Thus for any three points $s_{1}, s_{2}, s_{3}$ in $S$, some pair $\left(s_{i}, s_{j}\right)$ does not belong to $T(\mathscr{C}, \mathscr{D})$.

List in transfinite series $\mathscr{E}_{0} \mathscr{E}_{1} \ldots \mathscr{E}_{\alpha} \ldots \alpha<\kappa_{1}$ all c.g. sub- $\sigma$-algebras of $\mathscr{R}(S)$ proper in $\mathscr{B}(S)$. List also as $N_{0} N_{1} \ldots N_{\alpha} \ldots \alpha<\kappa_{1}$ all $m$-null members of $\mathscr{B}(S)$. We shall choose points $s_{\alpha}$ and $t_{\alpha}$ in $S$ via transfinite induction.

Assume that points $s_{\beta}, t_{\beta}$ have been chosen for $\beta<\alpha$. Remove a Borel subset of $S$ that is the union of the horizontal sections $\left[0,1\left[\times\left\{f\left(s_{\beta}\right)\right\}\right.\right.$ and $\left[0,1\left[\times\left\{f\left(t_{\beta}\right)\right\}\right.\right.$ all $\beta<\alpha)$ as well as the union $N_{0} \cup N_{1} \cup \ldots \cup N_{\alpha}$. This Borel set has $m$-measure zero. Denote what remains of $S$ by $R$.

Since $\mathscr{E}_{\alpha}$ is proper, we know that $\mathscr{E}_{\alpha}(R)$ is not separated. We choose points $s_{\alpha}, t_{\alpha}$ from $R$ not separated by $\mathscr{E}_{\alpha}$, but which are such, that the pair $\left(s_{\alpha}, t_{\alpha}\right)$ does not belong to $T(\mathscr{C}, \mathscr{D})$. We show presently that such a choice is possible:

1) If $\mathscr{E}_{\alpha}(R)$ contains an atom with at least three elements, then as mentioned above, two of these elements form a pair $(s, t)$ not in $T(\mathscr{C}, \mathscr{D})$. Put $s_{\alpha}=s$ and $t_{\alpha}=t$.

2) In this case, we assume that all $\mathscr{E}_{\alpha}(R)$-atoms are of cardinality $\leqslant 2$ and suppose that if $\{s, t\}$ is any $\mathscr{G}_{2}(R)$-atom, then $(s, t) \in T(\mathscr{C}, \mathscr{D})$. Let $W$ be the union of all $\mathscr{E}_{\alpha}(R)$-atoms of cardinality two (it follows from [6; p. 119] that $W$ is analytic). Then $A=W \cap A_{2}$ is an analytic subset of $] 0,1[\times] 0,1[$, each of whose horizontal sections is either singleton or empty. Thus there is a Borel set $B \supset A$ such, that $m(B)=0$. But since $\mathscr{E}_{\alpha}$ is proper in $\mathscr{B}$, we know that $\mathscr{E}_{\alpha}(R \backslash B) \neq \mathscr{B}(R \backslash B)$. This is the desired contradiction.

Having defined the points $s_{\alpha}, t_{\alpha}$ for each $\alpha<s_{1}$, put $X=\left\{s_{\alpha}, t_{\alpha}: \alpha<s_{1}\right\}$. Then $X$ is a Sierpiński subset of $S$ satisfying condition (J) but for which condition $(\mathrm{J}+)$ fails at $(\mathscr{C}, \mathscr{D})$.

Lemmas 4 and 12 imply that $m^{*}(X)=1$. It follows from Proposition 2 that $X$ is Blackwell, but not strongly Blackwell.

The space constructed in Proposition 18 yields an answer to an unsolved problem of D. Ramachandran: P930 in [14].

COROLLARY (CH). There is a probability space $(X, \mathscr{B}(X), P)$ whose Borel structure $\mathscr{B}(X)$ is Blackwell, but for which the notions of Kolmogoroff-and Steinhausindependence are not equivalent.

Remark. In [14] it is shown that the two definitions of independence are equivalent whenever $\mathscr{B}(X)$ is strongly Blackwell.

Proof. Let $X$ be the space constructed in the proof of Proposition 18 and put $P=m^{*}$. Let $h$ and $f$ be projection onto the first and second factor as functions defined on $X$. Then $h$ and $f$ are Steinhaus-independent random variables, meaning that

$$
\begin{aligned}
\mathscr{B}(h) & =\left\{h^{-1}(A): A \text { Borel }\right\}, \\
\mathscr{B}(f) & =\left\{f^{-1}(A): A \text { Borel }\right\}
\end{aligned}
$$

are $P$-independent $\sigma$-algebras. This follows from the fact that $m$ is a product measure on $S$ and from the equation $m^{*}(B \cap X)=m(B)$ for $B$ in $\mathscr{B}(S)$.

However, $h$ and $f$ are not Kolmogoroff-independent, meaning that

$$
\begin{aligned}
\mathscr{A}(h) & =\left\{h^{-1}(A): A \text { linear set, } h^{-1}(A) \in \mathscr{B}(X)\right\}, \\
\mathscr{A}(f) & =\left\{f^{-1}(A): A \text { linear set, } f^{-1}(A) \in \mathscr{B}(X)\right\} .
\end{aligned}
$$

are not $P$-independent. To see this, put $A=f\left(X \cap A_{1}\right)$. Then $A$ is a linear set with $f^{-1}(A)=X \cap A_{1}$ a member of $\mathscr{A}(f)$. Now $f^{-1}(A)=h^{-1}(0)$ and $P f^{-1}(A)$. 
$=P h^{-1}(0)=1 / 2$. This event is not independent of itself, so $\mathscr{A}(h)$ and $\mathscr{A}(f)$ are not independent.

$\S 7$. Products of Sierpiński sets. This section takes up the matter of when certain products of Sierpiński sets have the Blackwell property. In order to apply the techniques of Section 2, we begin by showing that for the $\sigma$-ideal $\mathscr{I}=\mathscr{I}(m)$, the notions of analytic and simple $\mathscr{I}$-density coincide.

Let $m$ be a Borel probability measure on a compact metric space $S$. Let $L(S)$ be the set of all Borel functions from $S$ to the interval [0,1]. For each $n \geqslant 1$, we define a set function $\iota_{n}$ on subsets of the product $S^{n}$. For any $R \subset S^{n}$, define

$$
\begin{aligned}
\iota_{n}(R)=\inf \left\{\int h_{1} d m+\ldots+\int h_{n} d m: h_{1}, \ldots, h_{n}\right. & \in L(S) \text { and } 1_{R}\left(s_{1}, \ldots, s_{n}\right) \\
& \left.\leqslant h_{1}\left(s_{1}\right)+\ldots+h_{n}\left(s_{n}\right), \text { all } s_{i} \in S\right\} .
\end{aligned}
$$

Here, $1_{R}$ is the indicator (characteristic) function of the set $R$. Hans Kellerer has considered this set function in his paper on the marginal problem [8]. (See 1.3 and 1.8 therein for an equivalent definition).

LEMMA 15. Let $m, S$, and $\iota_{n}$ be as described above.

1) The set function $\iota_{n}$ is a regular (Choquet) capacity on $S^{i}$.

2) $A$ subset $R$ of $S^{n}$ is $\mathscr{I}(m)$-reticulate if and only if $\iota_{n}(R)=0$.

Indications. Part 1 follows from 1.28 and 1.30 in [8]. Part 2 is established in [8] as part (b) in the proof of 1.15 .

LEMMA 16. Let $m$ be a Borel probability on a standard space $S$. For each $n \geqslant 1$, a subset $X$ of $S$ is anclytically $\mathscr{I}$-dense of order $n$ if and only if $X$ is $\mathscr{I}$-dense of order $n$.

Proof. One direction (only if) is immediate. For the other, we note that on analytic set $A \subset S^{n}$ is not $\mathscr{I}(m)$-reticulate if and only if there is some Borel $B \subset A$ which is not $\mathscr{I}(m)$-reticulate. This follows from Lemma 15 and the capacity theorem of Choquet. (If $S$ is topologized as a compact metric space, then $B$ may be chosen compact.)

Proposition 19. Let $m$ be a Borel probability on a standard space and let $X$ be an $m$-Sierpiniski subset of $S$ with $m^{*}(X)=1$. Let $A$ be an uncountable analytic subset of $S$. The following are equivalent:

1) $X^{n} \times S$ is strongly Blackwell;

2) $X^{n} \times A$ is strongly Blackwell;

3) $X^{n} \times A$ is Blackwell;

4) $X^{n} \times S$ is Blackwell;

5) $X$ is $\mathscr{I}(m)$-dense of order $2 n$ in $S$.

Indication. This re-expresses the corollary to Proposition 4, taking Lemma 16 into account.

The next set of results show that not all Sierpiński sets are created equal: some are "larger" than others. The larger ones enjoy more Blackwell properties.

Proposition 20. Let $m$ be a continuous Borel probability on a standard space $S$.
For each $n=1,2, \ldots$, there is a subset $Y$ of $S$ such that $Y$ is $\mathscr{I}(m)$-dense of order $n$, but not order $n+1$ in $S$.

Proof. We take $S$ to be the interval ]0, 1[ under its usual Borel structure. Let $1, f_{2}, \ldots$ be the Borel automorphisms of $S$ defined by $f_{k}(s)=s^{k}$. Let $\mathscr{J}$ be the $\sigma$-ideal of all countable subsets of $S$. The proof of Proposition 13 in [17] establishes the existence of a $Y \subset S$ such that

1) $Y$ is $\mathscr{J}$-dense of order $n$ in $S$;

2) $Y^{n+1}$ does not meet the subset

$$
\hat{G}=\left\{\left(s_{1}, \ldots, s_{n}, s_{n+1}\right): f_{1}\left(s_{1}\right)=\ldots=f_{n}\left(s_{n}\right)=f_{n+1}\left(s_{n+1}\right)\right\} \text { of } S^{n+1} .
$$

Property 1 implies that $Y$ is also $\mathscr{I}(m)$-dense of order $n$. It remains only to note that $G$ is not $\mathscr{I}(m)$-reticulate in $S^{n+1}$.

Corollary $1(\mathrm{CH})$. Let $m$ be a continuous Borel probability on a standard space $S$. For each $n=1,2, \ldots$, there is an $m$-Sierpinski subset $X$ of $S$ such that $X$ is $\mathscr{I}(m)$-dense fof order $n$, but not order $n+1$ in $S$.

Proof. Let $Y$ be as in Proposition 20 and apply Proposition 5. Note that if $Y$ is not dense of order $n+1$, then neither is any $X \subset Y$

Corollary $2(\mathrm{CH})$ Let $m$ be a continuous probability on a standard space $S$. For each $n=1,2, \ldots$, there is an $m$-Sierpiniski subset $X$ of $S$ such that

1) $X^{n} \times S$ is strongly Blackwell;

2) $X^{n+1} \times S$ is not Blackwell.

Proof. This follows from the preceeding corollary, combined with Proposition 19: take $S$ to be $\mathscr{I}(m)$-dense of order $2 n$, but not of any higher order.

$\S 8$. Selection theorems for $m$-threads and $m$-graphs. This section is devoted to establishing converses to parts B and $\mathrm{C}$ of Proposition 1 for the $\sigma$-ideal $\mathscr{I}(\mathrm{m})$ This will yield a complete characterization of Sierpiński sets with Blackwell properties and will facilitate a discussion of the combinatorial behavior of these sets.

Let $m$ be a Borel probability on a compact metric space $(S, d)$. Let $\mathscr{K}(S)$ denote space of all compact subsets of $S$ under the exponential topology of Hausdorff and Vietoris $[9 ; \mathrm{p} .160]$. The space $\mathscr{K}(S)$ is compact and is metrized by the Hausdorff metric [9; p. 214]. Let $C(S)$ denote the space of all continuous functions from $S$ to itself as a complete separable metric space under the uniform (supremum) norm. Finally, let $\mathscr{P}(S)$ be the space of all Borel probabilities on $S$. This space, under the weak topology induced by continuous real functions on $S$, is compact metric [12].

LEMMA 17. The real function $(K, v) \rightarrow v(K)$ is measurable (in fact, upper semicontinuous) on $\mathscr{K}(S) \times \mathscr{P}(S)$.

Proof. Given $\varepsilon \geqslant 0$ we prove that $\{(K, v): v(K) \geqslant \varepsilon\}$ is a closed set. Suppose that $\left(K_{n}, v_{n}\right) \rightarrow(K, v)$ as $n \rightarrow \infty$ with $v_{n}\left(K_{n}\right) \geqslant \varepsilon$ for each $n$. Fix $\delta>0$ and put

$$
K^{\delta}=\{x: d(x, u) \leqslant \delta \text { for some } u \text { in } K\}
$$


as the closed $\delta$-neighbourhood of $K$. Choose $n$ large so that $K_{n} \subset K^{\delta}$ and so that $v\left(K^{\delta}\right) \geqslant v_{n}\left(K^{\delta}\right)-\delta \geqslant v_{n}\left(K_{n}\right)-\delta \geqslant \varepsilon-\delta$. Letting $\delta \rightarrow 0$ gives $v(K) \geqslant \varepsilon$.

Let $f$ be a continuous real function on $S$. Let $m(p, \cdot)$ be a Markov transition kernel (disintegration) indexed by $p$ in $f(S)$ such that:

1) For each $p$ in $f(S)$, the set function $B \rightarrow m(p, B)$ is a Borel probability on $S$;

2) For each $B$ in $\mathscr{B}(S)$, the real function $p \rightarrow m(p, B)$ is Borel measurable on $f(S)$

3) For each $B$ in $\mathscr{B}(S)$, one may write

$$
m(B)=\int_{f(S)} m(p, B) d f(m)(p)
$$

4) For each $p$ in $f(S)$, one has $m\left(p, f^{-1}(p)\right)=1$.

For the existence of such kernels, see [13].

LEMMA 18. The mapping $p \rightarrow m(p, \cdot)$ is measurable from $f(S)$ into $\mathscr{P}(S)$. Proof. This follows immediately from the fact that the Borel structure on $\mathscr{P}(S)$ is generated by the evaluation functionals $\nu \rightarrow v(B)$ for $B$ ranging over $\mathscr{B}(S)$.

LEMMA 19. The maipping $p \rightarrow f^{-1}(p)$ is Borel mecisurcible from $f(S)$ to $\mathscr{K}(S)$.

Proof. See [9; p. 165].

LeMma 20. The set $\{(K, L): K \subset L\}$ is closed in $\mathscr{K}(S) \times \mathscr{K}(S)$.

Proof. See [9; p. 167].

LEMMA 21. The mapping $(K, L) \rightarrow K \times L$ is continuous from $\mathscr{K}(S) \times \mathscr{K}(S)$ to $\mathscr{K}(S \times S)$.

Proof. This follows from Remark 1 on p. 49 of [10] combined with the relation $\lim \left(K_{n} \times L_{n}\right)=\left(\lim K_{n}\right) \times\left(\lim L_{n}\right)$ in [9; p. 339].

LEMMA 22. Let $R$ be an $F_{\sigma}(\sigma$-compact) subset of $S \times S$. Then the set

$$
\mathscr{A}=\{p: R \text { is not } m(p, \cdot) \text {-reticulate }\}
$$

is a Borel subset of $f(S)$.

Proof. Write $R$ as the union of compact sets $R_{1} \cup R_{2} \cup \ldots=R$. Then we see that

$$
\mathscr{A}=\bigcup_{n=1}^{\infty}\left\{p: R_{n} \text { is not } m(p, \cdot) \text {-reticulate }\right\}
$$

so it suffices to work with a particular compact $R_{n}$. From Lemma 11 it follows that $R_{n}$ is $m(p, \cdot)$-reticulate if and only if $R_{n}^{e}$ contains rectangles with arbitrarily large sides for $m(p, \cdot)$. For each $k \geqslant 1$, define the following subset of $f(S) \times \mathscr{K}(S)$ :

$$
\mathscr{L}_{k}=\left\{(p, L): L \times L \subset R_{n}^{c} \text { and } m(p, L) \geqslant 1-1 / k\right\} .
$$

Lemma 17,18 and 21 imply that each $\mathscr{L}_{k}$ is Borel. Let $P\left(\mathscr{L}_{k}\right)$ be the (analytic) projection of $\mathscr{L}_{k}$ to $f(S)$. Then we may write

$$
\left\{p: R_{n} \text { is } m(p, \cdot) \text {-reticulate }\right\}
$$

as

$$
\bigcap_{k=1}^{\infty} P\left(\mathscr{L}_{k}\right)
$$

We have shown that the set $\mathscr{A}$ is co-analytic

Proposition 14 and Lemma 11 say that $R_{n}$ is not $m(p, \cdot)$-reticulate if and only if there is some probability $v$ on $S \times S$ with marginals $v_{1}=v_{2}=m(p, \cdot)$ such that $v\left(R_{n}\right)>0$. Thus we define the following subset of $f(S) \times \mathscr{P}(S \times S)$ :

$$
\mathscr{L}=\left\{(p, v): v_{1}=v_{2}=m(p, \cdot) \text { and } v\left(R_{n}\right)>0\right\} .
$$

Lemma 17 and 18 combined with the fact that the operations $v \rightarrow v_{1}$ and $v \rightarrow v_{2}$ are continuous show that $\mathscr{L}$ is a Borel set. Now $\left\{p: R_{n}\right.$ is not $m(p, \cdot)$-reticulate $\}$ is the projection of $\mathscr{L}$ to $f(S)$. Thus, $\mathscr{A}$ is an analytic set.

The set $\mathscr{A}$ is both co-analytic and analytic, and therefore Borel.

Define a subset $T_{0}$ of $S \times S$ by

$$
T_{0}=\left\{(s, t): f^{\prime}(s)=f(t)\right\} \text {. }
$$

Then $T_{0}$ is compact.

Proposition 21. For any $\sigma$-compact $\left(F_{\sigma}\right)$ subset $R$ of $T_{0}$, the following conditions are equivalent:

1) $R$ is m-reticulate;

2) $f(m)\{p: R$ is not $m(p, \cdot)$-reticulate $\}=0$.

Proof. Suppose that $R$ is $m$-reticulate, so that $R \subset(N \times S) \cup(S \times N)$ with $m(N)=0$. Then

$$
\int_{f(S)} m(p, N) d f(m)(p)=m(N)=0
$$

so that for $p$ outside some Borel subset $M$ of $f(S)$ with $f(m)(M)=0$, one has $m(p, N)=0$. For such $p$, clearly $R$ is $m(p, \cdot)$-reticulate. Condition 2 follows. Now assume that Condition 2 holds. Put $Q=\{p: R$ is $m(p, \cdot)$-reticulate $\}$. We know that $f(m)(Q)=1$. Fix $\varepsilon>0$. For each $p$ in $Q$, there is a compact $K \subset f^{-1}(p)$ with $K \times K \subset R^{c}$ and $m(p, K) \geqslant 1-\varepsilon$

Consider now the subset of $Q \times \mathscr{K}(S)$ defined by

$$
\mathscr{V}=\left\{(p, K): K \subset f^{-1}(p), K \times K \subset R^{c}, m(p, K) \geqslant 1-\varepsilon\right\} .
$$

Then from Lemmas $17-21$ it follows that $\mathscr{V}$ is a Borel subset of $Q \times \mathscr{K}(S)$, and so has a continuous selector on most of $Q$ : by the Jankov-von Neumann Theorem, has a continuous selector on most of continuous mapping $p \rightarrow K(p)$ defined on a compact subset $C$ of $Q$ such that

$$
\text { 1) } m(C) \geqslant 1-\varepsilon
$$

2) for each $p$ in $C$, the pair $(p, K(p))$ belongs to $\mathscr{V}$

The range of the mapping $p \rightarrow K(p)$ is compact, and so therefore is the union of all sets in its range, i.e.

$$
L=\bigcup_{p \in \mathcal{C}} K(p) .
$$


Also,

$$
m(L)=\int_{f(S)} m(p, L) d f(m)(p) \geqslant \int_{C} m(p, L) d f(m)(p),
$$

and since $K(p) \subset L$, one has $m(p, L) \geqslant m(p, K(p)) \geqslant 1-\varepsilon$. Therefore

$$
m(L) \geqslant(1-\varepsilon)(1-\varepsilon) \text {. }
$$

Now we claim that $L \times L \subset R^{c}$. Suppose there is some point $(x, y)$ in $(L \times L) \cap R$, Then, $(x, y) \in T_{0}$, so that $f(x)=f(y)$. Now there are points $p, q$ in $C$ with $x \in K(p) \subset f^{-1}(p)$ and $y \in K(q) \subset f^{-1}(q)$. This means that $p=q$. So $(x, y) \in$ $\in(K(p) \times K(p)) \cap R$, a contradiction from the definition of $\mathscr{r}$.

We have shown that for each $\varepsilon>0$, there is a Borel set $L$ with $m(L) \geqslant(1-\varepsilon)^{2}$ and such, that $L \times L \subset R^{c}$. It follows that $R$ is $m$-reticulate (Lemma 11).

We shall require the following technical

LEMMA 23. Let $f: K_{1} \rightarrow K_{2}$ be a continuous mapping between compact metric spaces $\left(K_{1}, d_{1}\right)$ and $\left(K_{2}, d_{2}\right)$. Then $f$ is one-one if and only if there is some countable dense subset $R$ of $K_{1}$ and a sequence of positive numbers $\varepsilon_{1} \varepsilon_{2} \varepsilon_{3} \ldots$ such that the following condition holds:

(*) whenever $r$ and $s$ are members of $R$ with $d_{1}(r, s) \geqslant 1 / n(n=1,2, \ldots)$, then $d_{2}(f(r), f(s))>\varepsilon_{n}$

Remark. The proof shows that if $f$ is one-one, then (*) holds for $R=K_{1}$.

Proof. Suppose that $f$ is indeed one-one on $K_{1}$. Then the inverse function $g=f^{-1}$ is uniformly continuous from $f\left(K_{1}\right)$ onto $K_{1}$. Thus for each $n \geqslant 1$ there is some positive $\varepsilon_{n}$ such that $d_{2}(u, v) \leqslant \varepsilon_{n}$ implies $d_{1}(g(u), g(v)) \leqslant 1 / n$ for all $u, v$ in $f\left(K_{1}\right)$. Rephrased, this means that whenever $d_{1}(x, y) \geqslant 1 / n$, then also $d_{2}(f(x), f(y))>\varepsilon_{n}$ for all $x, y$ in $K_{1}$.

Suppose that $f, R$, and $\varepsilon_{n}$ satisfy (*) and suppose for the sake of contradiction that there are points $x \neq y$ in $K_{1}$ with $f(x)=f(y)$. There is some $n \geqslant 1$ such that $d_{1}(x, y)>1 / n$. Also, there is some $\delta>0$ such that $d_{1}(x, u)<\delta$ implies $d_{2}(f(x), f(u))$ $<\varepsilon_{n} / 2$ for all $u$ in $K_{1}$ and such that $d_{1}(y, v)<\delta$ implies $d_{2}(f(y), f(v))<\varepsilon_{n} / 2$ for all $v$ in $K_{1}$. Choose points $r$ and $s$ in $R$ such that

1) $d_{1}(x, r)<\delta$

2) $d_{1}(y, s)<\delta$

3) $d_{1}(r, s)>1 / n$

Then

$$
d_{2}(f(r), f(s)) \leqslant d_{2}(f(r), f(x))+d_{2}(f(y), f(s))<\varepsilon_{n},
$$

a contradiction.

We now are in a position to prove our first major selection theorem. The insistence on 0 -dimensionality in the following result is for purely technical reasons (it enables one to extend continuous functions), and will not appear in the final product, which deals with the measurable case.
Proposition 22. Let $m$ be a Borel probability on a 0-dimensional compact metric space $S$. Suppose that $f$ is a continuous real function on $S$ generating the sub$\sigma$-algebra $\mathscr{C}$ of $\mathscr{B}(S)$. Suppose that $\mathscr{C}$ is $\mathscr{I}(m)$-proper in $\mathscr{B}(S)$. Then there is a homeomorphism $g$ between compact subsets of $S$ whose graph $K$ satisfies:

1) $K \subset\{(s, t): f(s)=f(t)$ and $s \neq t\}=T(\mathscr{C}, \mathscr{B})$

2) $K$ is a thread (not $m$-reticulate).

Proof. We shall define four analytic subsets $\mathscr{A}_{1}, \mathscr{A}_{2}, \mathscr{A}_{3}, \mathscr{A}_{4}$ of $f(S) \times$ $\times \mathscr{K}(S \times S)$. Then a selection theorem will be applied to their intersection much as in the proof of Proposition 21.

Define the set $\mathscr{A}_{1}$ by the rule

$$
\mathscr{A}_{1}=\left\{(p, K): K \subset f^{-1}(p) \times f^{-1}(p)\right\} .
$$

Lemmas 19,20 , and 21 show that $\mathscr{A}_{1}$ is closed. Likewise define

$$
\mathscr{A}_{2}=\{(p, K): K \cap \Delta=\varnothing\},
$$

where $\Delta$ is the diagonal in $S \times S$. The set $\mathscr{A}_{2}$ is open.

Define $\mathscr{A}$ to be the collection of all $(p, K)$ such that $K$ is not $m(p, \cdot)$-reticulate To see that $\mathscr{A}_{3}$ is analytic, we define a subset $\mathscr{E}_{1}$ of $f(S) \times \mathscr{K}(S \times S) \times \mathscr{P}(S \times S)$ by

$$
\mathscr{E}_{1}=\left\{(p, K, v): v(K)>0 \text { and } v_{1}=v_{2}=m(p, \cdot)\right\},
$$

where $v_{1}$ and $v_{2}$ are the univariate marginals of $v$. Then $\mathscr{E}_{1}$ is Borel and from Proposition 11 we see that $\mathscr{A}_{3}$ is the projection of $\mathscr{E}_{1}$ to $f(S) \times \mathscr{K}(S \times S)$.

The definition of $\mathscr{A}_{4}$ takes a few steps. When they are complete, $\mathscr{A}_{4}$ will be defined as the collection of all $(p, K)$ such that $K$ is the graph of a homeomorphism between compact subsets of $S$.

Consider the subset $\mathscr{E}_{2}$ of $\mathscr{K}(S) \times S^{\infty}$ consisting of all $\left(L, s_{1}, s_{2}, s_{3}, \ldots\right)$ such that $s_{1} s_{2} s_{3} \ldots$ is a sequence of points drawn from $L$ which is dense in $L$. Then $E_{2}$ is a $G_{\delta}$ set, since it may be written as the intersection of

$$
\bigcap_{n=1}^{\infty}\left\{\left(L, s_{1}, s_{2}, s_{3}, \ldots\right): s_{n} \in L\right\}
$$

which is closed, with

$$
\bigcap_{n=1}^{\infty}\left[\left\{\left(L, s_{1}, s_{2}, \ldots\right): U_{n} \cap L=\varnothing\right\} \cup \bigcup_{k=1}^{\infty}\left\{\left(L, s_{1}, s_{2}, \ldots\right): s_{k} \in U_{n}\right\}\right],
$$

where $U_{1} U_{2} \ldots$ is a fixed countable open base for the topology of $S$.

Define a subset $E_{3}$ of $\mathscr{C}(S) \times \mathscr{K}(S) \times S^{\infty} \times\left(\boldsymbol{R}^{+}\right)^{\infty}$ to be the intersection of the sets

$$
\left\{\left(f, L, s_{1}, s_{2}, \ldots ; \varepsilon_{1}, \varepsilon_{2}, \ldots\right): d\left(s_{i}, s_{j}\right) \leqslant 1 / n \text { or } d\left(f\left(s_{i}\right), f\left(s_{j}\right)\right)>\varepsilon_{n}\right\}
$$

as $n, i, j$ range over all positive integers. Then $\mathscr{E}_{3}$ is a $G_{\delta}$ set, as is $\mathscr{E}_{4}$, the intersection of $\mathscr{E}_{3}$ with $C(S) \times \mathscr{E}_{2} \times\left(\boldsymbol{R}^{+}\right)^{\infty}$ 
Define $\mathscr{E}_{5}$ to be the (analytic) projection of $\mathscr{E}_{4}$ to $C(S) \times \mathscr{K}(S)$. Then from Lemma $23, \mathscr{E}_{4}$ consists of all pairs $(f, L)$, where $f$ is a continuous function from $S$ to $S$ which is one-one on the compact set $L$.

Consider the mapping from $C(S) \times \mathscr{K}(S)$ to $\mathscr{K}(S \times S)$ sending $(f, L)$ to Graph $(f) \cap(L \times S)$. Following [9; p. 180] and [10; p. 70], as well as [3; p. 72], we see that the intersection operation $(K, L) \rightarrow K \cap L$ is measurable. From [9; p. 223], the same may be said of the operation $f \rightarrow \operatorname{Graph}(f)$. Thus the map in question is measurable. Define $\delta_{6}$ to be the image of $\mathscr{E}_{5}$ under this map; clearly, $\delta_{6}$ is an analytic set.

Finally, we may define $\mathscr{E}_{4}$ to be the product $f(S) \times \mathscr{E}_{6}$. As promised, $\mathscr{E}_{4}$ consists of all pairs $(p, K)$, where $K$ is the graph of some one-one continuous function (homeomorphism) defined on a compact subset $S$.

We have tacitly assumed that every such function may be extended continuously to all of $S$. Since $S$ is 0 -dimensional, this is guaranteed [9; p. 281]. Define $\mathscr{A}$ to be the intersection $\mathscr{A}=\mathscr{A}_{1} \cap \mathscr{A}_{2} \cap \mathscr{A}_{3} \cap \mathscr{A}_{4}$.

Consider now the set $T$ defined by

$$
T=T(\mathscr{C}, \mathscr{B})=\{(s, t): f(s)=f(t) \text { and } s \neq t\} .
$$

For each $p$ in $f(S)$, define the "cell" $T(p)$ as

$$
T(p)=\{(s, t): f(s)=f(t)=p \text { and } s \neq t\}
$$

Since $\mathscr{C}=\mathscr{B}(f)$ is proper in $\mathscr{B}(S)$, the set $T$ is not $m$-reticulate. Now $T$ is a $\sigma$-compact (open) subset of

$$
T_{0}=\{(s, t): f(s)=f(t)\},
$$

so that Proposition 21 applies with $R=T$. Put

$$
Q=\{p: T \text { is not } m(p, \cdot) \text {-reticulate }\} \text {. }
$$

Then

1) $m(Q)>0$;

2) for each $p$ in $Q$, the set $T(p)$ is not $m(p, \cdot)$-reticulate.

We shall now demonstrate that for each $p$ in $Q$, there is some $K$ in $\mathscr{K}(S \times S)$ such that $(p, K) \in \mathscr{A}$. So fix $p \in Q$.

Case 1 . The measure $m(p, \cdot)$ is purely discrete, We see that $m(p, \cdot)$ cannot be a Dirac mass concentrated at a single point $x$ in $f^{-1}(p)$, since $T(p)$ is not $m(p, \cdot)$-reticulate. So there must be two points $x_{1}$ and $x_{2}$ in $f^{-1}(p)$ with $m\left(p, x_{1}\right)$ and $m\left(p, x_{2}\right)$ positive. Then put $K=\left\{\left(x_{1}, x_{2}\right)\right\}$.

Clearly, $(p, K)$ belongs to $\mathscr{A}$, since

1) $K \subset f^{-1}(p) \times f^{-1}(p)$;

2) $K \cap \Delta=\varnothing$

3) $K$ is not $m(p, \cdot)$-reticulate;

4) $K$ is the graph of a one-one continuous function between compact (singleton) subsets of $S$.
Case 2. The measure $m(p, \cdot)$ is not discrete, so there exists some Borel set $A \subset f^{-1}(p)$ with $m(p, A)>0$ and on which $m(p, \cdot)$ is continuous. Let $n$ denote the measure $m(p, \cdot)$ restricted to subsets of $A$. By the usual isomorphism tricks, there is a Borel-isomorphism $k$ of $A$ onto a real interval $J$ taking $n$ to Lebesgue measure $k(n)$ on $J$. Then $(s, t) \rightarrow(k(s), k(t))$ takes $A \times A$ isomorphically onto $J \times J$ and sends $(A \times A) \cap \Delta$ to the diagonal $\Delta_{0}$ of $J \times J$.

Now $(J \times J) \backslash \Delta_{0}$ contains the graph of a Borel automorphism $h$ of $J$ onto itself which preserves the linear measure $k(n)$. Then the composition $g=k^{-1} \circ h \circ k$ is an automorphism of $A$ onto itself preserving the measure $n$ such that graph $(g)$ $\subset(A \times A) \backslash A$. By Lusin's theorem, there is a compact $L \subset A$ with $n(L)>0$ on which $g$ is continuous (a homeomorphism).

Define $K$ to be the graph of the restriction of $g$ to the set $L$. Then $(p, K)$ belongs to $\mathscr{A}$, since
1) $K \subset f^{-1}(p) \times f^{-1}(p)$;
2) $K \cap \Delta=\varnothing$;
3) $K$ is not $m(p, \cdot)$-reticulate;

4) $K$ is the graph of a one-one continuous mapping between compact subsets of $S$.

Remark. The above reasoning shows that $Q=\{p: m(p, \cdot)$ is not a point measure\}.

We have established that for each $p$ in $Q$, there is some $K$ in $\mathscr{K}(S \times S)$ with $(p, K) \in \mathscr{A}$. An application of the Jankov-von Neumann selection theorem, combined with Lusin's theorem produces a continuous selector for $\mathscr{A}$ over most of $Q$ : there is a continuous mapping $p \rightarrow K(p)$ defined on a compact subset $C$ of $Q$ such that

1) $m(C)>0$;

2) for each $p$ in $C$, the pair $(p, K(p))$ belongs to $\mathscr{A}$.

The range of the mapping $p \rightarrow K(p)$ is compact, and so therefore is the union of all sets in that range, i.e.

$$
K=\bigcup_{p \in C} K(p)
$$

Now for $p \in C$, the set $K(p)$ is not $m(p, \cdot)$-reticulate. By Proposition 21 , it follows that $K$ is not $m$-reticulate. Clearly, $K$ is the graph of a one-one function. Since $K$ is compact, this function is a homeomorphism $g$. Lastly, we note that $K \subset T$.

The measurable case easily reduces to the continuous one described in Proposition 22 .

Propositron 23. Let $m$ be a Borel probability on a standard space $S$. Suppose that $f$ is a measurable real function on $S$ generating the sub- $\sigma$-algebra $\mathscr{C}$ of $\mathscr{B}(S)$. Suppose that $\mathscr{C}$ is proper in $\mathscr{B}(S)$. Then there is a thread $K$ contained in $T(\mathscr{C}, \mathscr{B})$ $=\{(s, t): f(s)=f(t)$ and $s \neq t\}$.

3 - r'undamenta Mathematicae 127.1 
Proof. We assume that $S$ has been metrized as a 0 -dimensional compact metric space. The condition that $\mathscr{C}$ be proper in $\mathscr{B}(S)$ is equivalent to the statement that $T=T(\mathscr{C}, \mathscr{B})$ is not reticulate. From Lemma 11 , there is some $\varepsilon>0$ such that for any $B$ in $\mathscr{B}(S)$ with $m(B) \geqslant 1-\varepsilon$, the set $(B \times B) \cap T$ is not reticulate.

Keeping $\varepsilon$ as specified and applying Lusin's theorem to $f$, we obtain a compact $S_{0} \subset S$ such that

1) $m\left(S_{0}\right) \geqslant 1-\varepsilon$

2) $f$ is continuous on $S_{0}$.

Then define a probability $m_{0}$ on $S_{0}$ by the rule

$$
m_{0}(B)=m\left(B \mid S_{0}\right)=\frac{m\left(B \cap S_{0}\right)}{m\left(S_{0}\right)}
$$

Let $f_{0}$ be the restriction of $f$ to $S_{0}$. Then Proposition 22 may be applied to $m_{0}, S_{0}$, and $f_{0}$, noting that $\mathscr{B}\left(S_{0}\right)$ is $m_{0}$-proper in $\mathscr{B}\left(S_{0}\right)$ and that if a set $K \subset S_{0} \times S_{0}$ is not $m_{0}$-reticulate, then $R$ is certainly not $m$-reticulate.

The techniques we have developed enable us to prove the following selection theorem. The argument is similar to the one for Proposition 22, with a couple of subtle differences. These differences turn out to mark the distinction between the Blackwell and strong Blackwell properties for Sierpiński sets.

Proposition 24. Let $m$ be a Borel probability on a 0-dimensional compact metric space $S$. Suppose that $f$ and $g$ are continuous real functions on $S$ generating sub- $\sigma$-algebras $\mathscr{C} \subset \mathscr{D}$ in $\mathscr{B}(S)$ with $\mathscr{C}$ m-proper in $\mathscr{D}$.

Then there is a contimuous mapping $k$ between compact subsets of $S$ whose graph $K$ satisfies:

1) $K \subset\{(s, t): f(s)=f(t)$ cind $g(s) \neq g(t)\}=T(\mathscr{C}, \mathscr{D})$;

2) $K$ is not $m$-reticulate ( $K$ is an $\mathscr{I}(m)$-graph).

Proof. Let $m(p, \cdot)$ be a transition kernel indexed by $p$ in $f(S)$ just as above. Note that $\mathscr{C}$ proper in $\mathscr{D}$ means that $T(\mathscr{C}, \mathscr{D})$ is not $m$-reticulate.

Following the proof of Proposition 22, we define four analytic subsets $\mathscr{A}_{1}, \mathscr{A}_{2}, \mathscr{A}_{3}, \mathscr{A}_{4}$ of $f(S) \times \mathscr{K}(S \times S)$. The set $\mathscr{A}_{1}$ is defined as

$$
\mathscr{A}_{1}=\left\{(p, K): K \subset f^{-1}(p) \times f^{-1}(p)\right\} .
$$

Let $D$ be the closed set

$$
D=\{(s, t): g(s)=g(t)\}
$$

and define

$$
\mathscr{A}_{2}=\{(p, K): K \cap D=\varnothing\} .
$$

Then $\mathscr{A}_{1}$ is closed, and $\mathscr{A}_{2}$ is open.

Let $\mathscr{A}_{3}$ be the collection of all $(p, K)$ such that $K$ is not $m(p, \cdot)$-reticulate. Lastly, $\mathscr{A}_{4}$ is defined as the collection of all $(p, K)$ such that $K$ is the graph of a continuous mapping between compact subsets of $S$. As in the proof of Proposition 22, the sets $\mathscr{A}_{3}$ and $\mathscr{A}_{4}$ are analytic. The set $\mathscr{A}$ is defined to be the intersection $\mathscr{A}=\mathscr{A}_{1} \cap \mathscr{A}_{2} \cap \mathscr{A}_{3} \cap \mathscr{A}_{4}$.

For each $p$ in $f(S)$, define the "cell" $T(p)$ as

$$
T(p)=\{(s, t): f(s)=f(t)=p \text { and } g(s) \neq g(t)\} .
$$

Applying Lemma 22 to $T(\mathscr{C}, \mathscr{D})$, we produce the Borel set

$$
Q=\{p: T(\mathscr{C}, \mathscr{D}) \text { is not } m(p, \cdot) \text {-reticulate }\}
$$

with the properties

1) $m(Q)>0$;

2) for each $p \in Q$, the set $T(p)$ is not $m(p, \cdot)$-reticulate.

We now demonstrate that for each $p$ in $Q$, there is some $K$ in $\mathscr{K}(S \times S)$ with $(p, K) \in \mathscr{A}$. So fix $p \in Q$.

Fact. The measure $m(p, \cdot)$ cannot be entirely concentrated on a single $\mathscr{D}$-atom $g^{-1}(q)$. Were it so, then $g^{-1}(q) \times g^{-1}(q) \subset(S \times S) \backslash T(p)$ would contradict property 2 supra.

Case 1. There is some point $y$ in $f^{-1}(p)$ with $m(p, y)>0$. Define $q=g(y)$ and let $L$ be a compact subset of $f^{-1}(p) \backslash q^{-1}(q)$ with $m(p, L)>0$. (We have used the Fact.) Put $K=L \times\{y\}$. Then $(p, K) \in \mathscr{A}$.

Case 2. The measure $m(p, \cdot)$ is continuous. Define $g_{0}$ to be the restriction of $g$ to the set $f^{-1}(p)$. Let $n$ be the image of the measure $m(p, \cdot)$ under the map $g_{0}$. By the Fact, $n$ is not a simple point mass. Thus there is some linear Borel set $A$ with $0<n A<1$. Put $B=g_{0}^{-1}(A)$ and $B^{\prime}=f^{-1}(p) \backslash B$. Then $m(p, B) m\left(p, G^{\prime}\right)>0$, so that $B \times B^{\prime}$ is a Borel subset of $T(p)$ containing a compact $\mathscr{F}(m(p, \cdot))$-thread $K$, as desired.

Remark. It is instructive to carry out the decomposition of $T(\mathscr{C}, \mathscr{D})$ into the sets $T(p)$ as well as the corresponding disintegration of $m$ for the example constructed in Proposition 18. The essential difference between conditions $(J)$ and $(J+)$ then becomes quite vivid.

We have established that for each $p$ in $Q$, there is some $K$ in $\mathscr{K}(S \times S)$ with $(p, K) \in \mathscr{A}$. An application of the Jankov-von Neumann selection theorem, combined with Lusin's theorem, produces a continuous selector for $A$ over most of $Q$ : there is a continuous mapping $p \rightarrow K(p)$ defined on a compact subset $C$ of $Q$ such that

1) $m(C)>0$;

2) for each $p$ in $C$, the pair $(p, K(p))$ belongs to $\mathscr{A}$.

The range of the mapping $p \rightarrow K(p)$ is compact, and so therefore is the union of all sets in its range, i.e.

$$
K=\bigcup_{p \in C} K(p)
$$

Now for $p \in C$, the set $K(p)$ is not $m(p, \cdot)$-reticulate. By Proposition 21, it follows that $K$ is not $m$-reticulate. Clearly, $K$ is the graph of some function $k$. Since $K$ is compact, $k$ is continuous. Finally, we note that $K \subset T(\mathscr{C}, \mathscr{D})$. 
As before, we pass to the measurable case. The proof of the following theorem is entirely analogous to that of Proposition 23.

Proposition 25. Let $m$ be a Borel probability on a standard space $S$. Suppose that $f$ and $g$ cire medisurable recil functions on $S$ generating the sub- $\sigma$-algebras $\mathscr{B} \subset \mathscr{D}$ in $\mathscr{B}(S)$ with $\mathscr{C} m$-proper in $\mathscr{D}$. Then there is an $\mathscr{I}(m)$-graph $K$ contained in

$$
T(\mathscr{B}, \mathscr{D})=\{(s, t): f(s)=f(t) \text { and } g(s) \neq g(t)\} \text {. }
$$

It is now possible to obtain a complete characterization of Sierpiński sets with Blackwell and strong Blackwell properties.

Proposition 26. Let $m$ be a Borel probability on a standard space $S$. Suppose that $X$ is $\mathscr{I}(m)$-Lusin in $S$ with $m^{*}(X)=1$. Then the following are equivalent:

1) $X$ is a Blackwell set;

2) $X$ satisfics condition $(\mathrm{J})$;

3) $X \times X$ mcets every $\mathscr{I}(m)$-thread in $S \times S$.

Also, the following are equivalent:

4) $X$ is strongly Blackwell;

5) $X$ satisfies condition $(\mathbf{J}+)$;

6) $X \times X$ meets every $\mathscr{I}(\mathrm{m})$-grciph in $S \times S$

Proof. The equivalences $1 \Leftrightarrow 2$ and $4 \Leftrightarrow 5$ follow from Proposition 1 . The implications $2 \Leftrightarrow 3$ and $5 \Leftrightarrow 6$ follow from Proposition 2. Lastly, the implications $3 \Leftrightarrow 2$ and $6 \Leftrightarrow 5$ are easily deduced from Propositions 23 and 25 , respectively.

§ 9. Direct sums of Sierpiński sets. Suppose $X$ is a subset of the standard space $S$ and that $\mathscr{I}$ is a $\sigma$-ideal in $\mathscr{B}(S)$. Let $N$ be an arbitrary, nonvoid, countable set (finite or infinite). If $N$ is given the discrete Borel structure, then $X \times N$ is a direct union of copies of $X$ contained in the standard space $S \times N$. Define a $\sigma$-ideal $\mathscr{J}$ in $\mathscr{B}(S \times N)$ to be the collection of all countable unions of sets of the form $M \times\{n\}$, where $M \in \mathscr{I}$ and ${ }^{\prime} n \in N$.

Lemma 24. If $X$ is $\mathscr{I}$-dense in $S$, then $X \times N$ is $\mathscr{J}$-dense in $S \times N$. If $X$ is an I-Lusin set, then $X \times N$ is a f-Lusin set in $S \times N$.

Proof. Immediate.

Proposition 27. Let $m$ be a Borel probability on a standard spaice $S$ cind suppose that $X$ is an $m$-Sierpinski subset of $S$.

1) If $X$ is Blackwell, then so is $S \times N$.

2) If $X$ is strong Blackwell, then so is $S \times N$.

Proof. Without loss of generality, one may assume that $m^{*}(X)=1$. We establish 2) then indicate the (similar) proof of 1).

Let $d$ be any probability on $N$ with $d(n)>0$ for each $n$ in $N$. Put $m^{\prime}=m \otimes d$ on $S \times N$. Then the $\sigma$ tideal $\mathscr{J}$ discussed above consists of the null sets for $m^{\prime}$. Lemma 24 implies that $X \times N$ is an $m^{\prime}$-Sierpiński sèt. From Proposition $1, X \times X$ meets every $\mathscr{I}-(m)$-graph in $S \times S$. It is easily seen that $(X \times N) \times(X \times N)$ intersects every $m^{\prime}$-graph in $(S \times N) \times(S \times N)$. Proposition 26 implies that $X \times N$ is strong Blackwell.

The same proof applies to Condition 1, with "graph" replaced by "thread".

A forthcoming paper by the first author will contain a proof that whenever $X$ is a Blackwell space, then so too is $X \times N$. The situation for strong Blackwell spaces is still unresolved.

$\S$ 10. A conjecture about $m$-graphs. The preceeding analysis has not answered the question of whether $X \times S$ is Blackwell whenever $X$ is a strong Blackwell Sierpiński set. However, it will be shown that this matter reduces to a conjecture about measurable selections, one that is of some independent interest.

Specifically, we have not been able to decide the following statements:

HYPOTHESIS (G). Let $m$ be a continuous Borel probability on a standard space $S$. If a set $B$ in $\mathscr{B}\left(S^{2}\right)$ is not $\mathscr{I}(m)$-reticulate in $S^{2}$, then $B$ contains an $\mathscr{I}(m)$-graph.

HYPOTHESIS $\left(\mathrm{G}^{\prime}\right)$. Let $m$ be a continuous Borel probability on a standard space $S$. If $Y$ is a subset of $S$ such that $Y \times Y$ meets every $\mathscr{I}(m)$-graph, then $Y$ is $\mathscr{I}(m)$-dense of order 2.

Proposition 28. Hypothesis (G) implics hypothesis $\left(\mathrm{G}^{\prime}\right)$. Under $\mathrm{CH}$, the converse is also true.

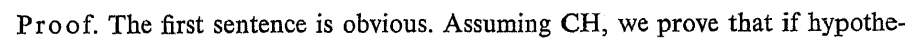
sis $(\mathrm{G})$ fails, then so does hypothesis $\left(\mathrm{G}^{\prime}\right)$. Suppose that $B \in \mathscr{B}\left(S^{2}\right)$ is not $\mathscr{I}(m)$-reticulate and contains no $\mathscr{I}(m)$-graphs. From Lemma $3,(m \otimes m)(B)=0$. By Fubini's Theorem, we may assume that for each $s \in S$, the sections

$$
\begin{aligned}
& B_{s}=\{t \in S:(s, t) \in B\}, \\
& B^{s}=\{t \in S:(t, s) \in B\}
\end{aligned}
$$

are of $m$-measure zero. (We may remove the other sections and preserve the character of $B$.) We also assume that $B$ is symmetric about the line $y=x$. (We may replace $B$ with its symmetrization $B \cup B^{1}$, where $B^{1}=\{(t, s):(s, t) \in B\}$.)

Well-order the collection of all $\mathscr{I}(m)$-graphs as $G_{0} G_{1} G_{2} \ldots G_{\alpha} \ldots, \alpha<\aleph_{1}$, and choose points $y_{1}^{(\alpha)}, y_{2}^{(\alpha)}, \alpha<\aleph_{1}$, according to the following scheme: $\left(y_{1}^{(0)}, y_{2}^{(0)}\right) \in G_{0} \backslash B$; for each $\alpha$, define $Z_{\alpha}$ to be the set of all $s \in S$ such that either $\left(s, y_{1}^{(\alpha)}\right) \in B$ of $\left(s, y_{2}^{(\alpha)}\right) \in B$. Put $Y_{\alpha}=\bigcup\left\{Z_{\beta}: \beta<\alpha\right\}$ and note that $m\left(Y_{\alpha}\right)=0$. In general, we select $\left(y_{1}^{(\alpha)}, y_{2}^{(\alpha)}\right) \in G_{\alpha} \backslash\left(B \cup\left\langle Y_{\alpha}, Y_{\alpha}\right\rangle\right)$.

Put $Y=\left\{y_{1}^{(\alpha)}, y_{2}^{(\alpha)}: \alpha<\aleph_{1}\right\}$. The set $Y \times Y$ meets each of the $\mathscr{I}(m)$-graphs $G_{\psi}$, but $(Y \times Y) \cap B$ is void.

The importance of hypothesis $(G)$ for the present analysis lies in the following equivalence. It provides a partial answer to problem (P8) in [1], at least for Sierpiński sets.

Proposition 29. Let $S$ be an uncountable standard space and consider the following statements:

1) Hypothesis (G); 
2) Suppose $m$ is a continuous probability on $\mathscr{B}(S)$ and that $X \subset S$ is an $m$-Sierpiński set. If $X$ is strongly Blackwell, then so also is $X \times S$.

Statement 1 implies statement 2 . Under $\mathrm{CH}$, they are cquivalent.

Proof. $1 \Rightarrow 2$. Assume that $X \subset S$ is a strongly Blackwell $m$-Sierpiński set. It is no loss of generality to assume that $m^{*}(X)=1$. By Proposition $2, X \times X$ intersects every $\mathscr{I}(m)$-graph in $S \times S$. Hypothesis (G) implies hypothesis ( $\left.\mathrm{G}^{\prime}\right)$, so that $X$ is $\mathscr{I}(m)$-dense of order 2. Proposition 1 then implies that $X \times S$ is strongly Blackwell.

$2 \Rightarrow(\mathrm{CH})$. Suppose hypothesis $(\mathrm{G})$ fails. Then by Proposition 28 , so does hypothesis $\left(G^{\prime}\right)$. Thus there is some $Y \subset S$ such that $Y \times Y$ meets every $\mathscr{I}(m)$-graph and such that $Y$ is not $\mathscr{I}(m)$-dense of order 2. From Proposition 5, we see that there is an $m$-Sierpiński set $X \subset Y$ with the same properties. Then $X$ is strongly Blackwell, but $X \times S$ is not a Blackwell space (Proposition 19).

Hypothesis (G) seems to be related to the analysis of the so-called "doubly stochastic measures" (vide [11]). Not-(G) implies that there is an extreme doublystochastic measure not concentrated on any countable union of measurable graphs. An example of such a measure is known to exist [11], so this implication yields nothing new, and its proof (using the Choquet-Bishop-de Leeuw theorem on integral representations) is therefore omitted. It may be taken as very mild evidence that $(G)$ is false.

\section{References}

[1] K.P. S. Bhaskara Rao and B. V. Rao, Borel spaces, Diss. Math. 190 (1981).

[2] J. B. Brown and G. V. Cox, Classical theory of totally imperfect spaces, Real Anal. Exchange 7 (1981-2), 185-232.

[3] J. P. R. Christensen, Topology and Borel Structure, North-Holland Publishing Company, Amsterdam 1974.

[4] D. Fremlin, On Blackwell algebras (unpublished manuscript, 1980).

[5] S. Graf and R. D. Mauldin, Measurable one-one selections and transition kernels, Amer. J. Math. 107 (1985), 407-425.

[6] J. Hoffmann-Jørgensen, The Theory of Analytic Spaces, Various Publ. Ser., No. 10 Aarhus Universitet (1970).

[7] J. Jasiniski, On the Blackwell property of Lusin sets, Proc. Amer. Math. Soc. 95 (1985), 303-306.

[8] H. G. Kellerer, Duality theorems for marginal problems, Z. Wahrsch 67 (1984), 399-432.

[9] K. Kuratowski, Topology I, Academic Press-PWN, New York-Warsaw 1966

[10] - Topology II, Academic Press-PWN, New York-Warsaw 1968.

[11] V. Losert, Counter-examples to some conjectures about doubly stochastic measures, Pacific J. Math. 99 (1982), 387-397.

[12] K. R. Parthasarathy, Probability Measures on Metric Spaces, Academic Press, New York 1967.

[13] D. Ramachandran, Perfect Measures, Macmillan India, Delhi 1979.

[14] - On the two definitions of independence, Colloq. Math. 32 (1975), 227-31.

[15] H. Sarbadhikari, A note on reticulated sets, pre-print.
[16] R. M. Shortt, Strassen's marginal problem in two or more dimensions, Z. Wahrsch. 64 (1983), 313-325.

[17] -, Borel-density, the marginal problem, and isomorphism types of analytic sets, Pacific J. Math. 113 (1984), 183-200.

[18] - Borel-dense Blackwell spaces are strongly Blackwell (pre-print).

[19] - Reticulated sets and the isomorphism of analytic powers, Pacific J. Math. 119 (1985), 215-226.

[20] W. Sierpiński, Hypothèse du Continu, 2nd edition, Chelsea, New York 1956.

[21] V. Strassen, Probability measures with given marginals, Ann. Math. Stat. 36 (1965), 423-439.

R. M. Shortt

DEPARTMENT OF MATHEMATICS

MICHIGAN TECHNOL.OGY UNIVERSITY

Houghton, Michigan 49931 